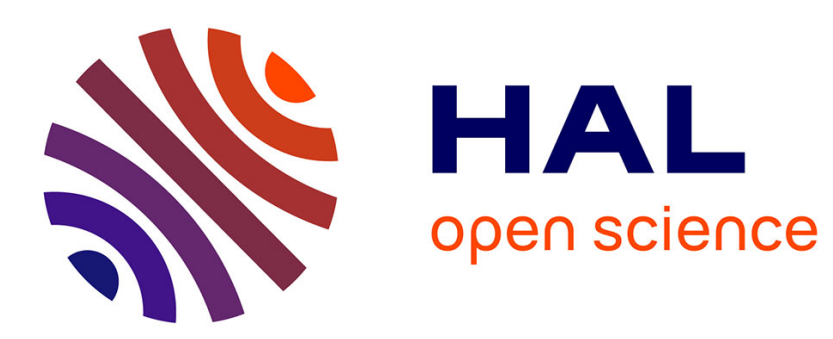

\title{
Modeling Granular Materials: Century-Long Research across Scales
}

\author{
Farhang Radjai, Jean-Noël Roux, Ali Daouadji
}

\section{To cite this version:}

Farhang Radjai, Jean-Noël Roux, Ali Daouadji. Modeling Granular Materials: Century-Long Research across Scales. Journal of Engineering Mechanics - ASCE, 2017, 143 (4), 10.1061/(ASCE)EM.19437889.0001196 . hal-01699485

\section{HAL Id: hal-01699485 \\ https://hal.science/hal-01699485}

Submitted on 2 Feb 2018

HAL is a multi-disciplinary open access archive for the deposit and dissemination of scientific research documents, whether they are published or not. The documents may come from teaching and research institutions in France or abroad, or from public or private research centers.
L'archive ouverte pluridisciplinaire HAL, est destinée au dépôt et à la diffusion de documents scientifiques de niveau recherche, publiés ou non, émanant des établissements d'enseignement et de recherche français ou étrangers, des laboratoires publics ou privés. 


\title{
MODELING GRANULAR MATERIALS: CENTURY-LONG RESEARCH ACROSS SCALES
}

\author{
Farhang Radjai ${ }^{1}$ \\ Jean-Noël Roux ${ }^{2}$
}

Ali Daouadji ${ }^{3}$

\begin{abstract}
Granular materials are the most recurrent form of solid-state matter on Earth. They challenge researchers and engineers in various fields not only because they occur with a broad variety of grain sizes, shapes and interactions in nature and industry, but also because they show a rich panoply of mechanical states. Despite this polymorphism, all these different types of soils, powders, granules, ores, pharmaceutical products, .... are instances of the granular matter with the same least common denominator of being sandlike (psammoid in Greek), i.e. solid grains interacting via frictional contacts. This review describes milestone contributions to the field of granular materials since the early elastic-plastic models developed for soils in the 1950s. The research on granular materials has grown into a vast multi-disciplinary field in the 1980s with increasing focus on the microstructure and owing to new experimental tools and discrete simulation methods. It turns out that the granular texture, particle-scale kinematics and force transmission are far more complex than presumed in early micromechanical models of granular materials. Hence, constitutive relations cannot easily be derived from the particle-scale behavior although advanced continuum models have been developed to account for anisotropy, intermediate stress and complex loading paths. The subtle elastic properties and origins of bulk friction will be discussed, as well as the effects of particle shape and size distribution. The review covers also recent developments in macroscopic modeling such as the thermomechanical approach, anisotropic critical state theory, nonlocal modeling approach, inertial flows and material instabilities. Finally, a brief account is given of open issues and some new frontiers and challenges in the field.
\end{abstract}

Keywords: granular materials, constitutive modeling, micromechanics, multiscale modeling, thermomechanics, gradient plasticity, hypoplasticity, yield function, fabric tensor, flow rule, shear band, state parameter, non-associated flow rule, critical state theory, internal friction, dilatancy, force 
chains, fabric tensor, nonaffine velocity, nonlocal model, jamming, entropy, second-order work, discrete element method

\section{INTRODUCTION: AN OVERVIEW}

Granular matter has been a source of inspiration as much to little builders of sand castles on the beach as to philosophers and poets who have been seeking in grains a window opening to the invisible secrets of matter and life. "To See a World in a Grain of Sand ...", wrote William Blake. Modern physics tells us that, depending on the resolution of measurement devices, a single grain contains almost all about the laws of nature at increasingly smaller scales. But at larger scales, those grains hold also the secrets of granular matter. Dissipative interactions among grains give rise to an extraordinarily rich and complex bulk behavior of an assembly of grains. Eminent figures of science and engineering were deeply concerned about odd behaviors arising from those interactions. The unique properties of friction between two solid grains and its consequence for the stability of a granular talus were introduced by Charles Coulomb (Coulomb 1773; Coulomb 1781). Dilatancy (volume change under shear), discovered by Osborne Reynolds, appeared as much counterintuitive as friction (Reynolds 1885). Memory effects, or "historical element" as put by James Maxwell, were also recognized as a distinguishing feature of granular materials (Darwin 1883). The grains can move like molecules in a gas but they dissipate energy by inelastic collisions and can get "jammed" in a variety of configurations allowed by friction between grains and the action of their weights or a confining stress. Terzaghi was intrigued by the variability of soils under different loading conditions and the quasi-impossibility of arriving at the same level of scientific reliability as in other materials (Terzaghi 1943). In a similar vein, as a result of energy dissipation at the grain scale, granular materials do not seem to fit the general principles upon which the statistical mechanics and thermodynamics of molecular fluids and solids were built (Jaeger and Nagel 1996).

There can be no comprehensive review of research on all aspects of granular matter in a single paper - even when limited to cohesionless dry granular materials as in the present paper. Here, the focus will be on those features that are believed to have strong bearing on the future developments in this field. It should also be borne in mind that, despite many multidisciplinary workshops organized for 30 years, there still remain high cultural barriers between various communities involved in research on granular materials. The level of expectation from such a research and the ultimate 
goals are often quite different. Our goal here is to contribute to cross fertilization among disciplines by mainly emphasizing the guiding ideas and concepts and avoiding technical developments.

During the $20^{\text {th }}$ century, two scientific communities were involved in modeling granular materials: soil mechanics and powder technology. The Mohr-Coulomb plastic behavior is and remains at the heart of soil mechanics which has been primarily interested in predicting soil failure whereas powder technology has mostly been concerned with large deformations and continuous flow of granular materials often regarded as a suspension. In the $10^{\text {th }}$ Rankine Lecture in 1970, Roscoe emphasized the need to understand the stress-strain behavior of soils well before failure under complex loading conditions as encountered in engineering practice (Roscoe 1970). Fully aware of the need for a fundamental approach, he indicated the route towards a fundamental understanding of soils by working "with soils in their simplest possible states (e.g. well graded sands and saturated remolded clays) so that their properties can be defined by the minimum possible number of parameters", preparing "soil samples in initially uniform states", developing test equipments and "non-destructive methods of checking the uniformity of the behavior of the soils at all stages of these tests" and developing "scanning electron microscopy methods of studying the change of soil fabric during mechanical deformation". This is the route which was followed during the coming decades.

A consistent model of soil plasticity was actually achieved through the "critical-state soil mechanics" of the Cambridge School (Roscoe et al. 1958; Schofield and Wroth 1968; Wood 1990). By accounting for both frictional and volume-change behaviors of soils and, more importantly, by recognizing a family of memoryless states reached after long enough shearing, it provided for the first time a general framework for the quasi-static behavior of both clays and granular soils. The critical state theory is the core of nearly all constitutive models that were developed later to account for complex loadings paths. Critical states are asymptotic states approached for large enough strains, applied monotonically and quasistatically. Constitutive laws predicting this gradual approach are traditionally elastoplastic in nature. The elastic properties of granular materials have been clarified, over the past 25 years, thanks to improved experimental techniques apt to measure very small strain intervals (Shibuya et al. 1992; Hicher 1996). Elastic waves (Goddard 1990a) are actually the reflection of the quasistatic elastic behavior of small amplitude perturbations about an equilibrium state of a solid granular assembly (Thomann and Hryciw 1990; Shibuya et al. 1992; Geoffroy et al. 
2003). Due to nonlinear contact elasticity, their velocity depends on the stress level. While grainscale disorder induces incoherent propagation, larger wavelengths propagate coherently (Liu and Nagel 1992; Liu 1994; Liu and Nagel 1994; Jia et al. 1999).

Micromechanical approaches to the plastic (Christoffersen et al. 1981; Bathurst and Rothenburg 1988; Chang and Misra 1990) and elastic (Walton 1987; La Ragione and Jenkins 2007) behaviors of granular materials have been an active area from the outset of modern research on granular materials. The onset of instabilities in granular materials as a function of the loading program has also been a subject of extensive investigation under homogeneous boundary conditions (Vardoulakis 1979; Lade 1994; Vardoulakis and Sulem 1995; Nova 1994; Darve and Laouafa 1999; Lade 2002; Nicot and Darve 2007; Chang et al. 2011). Directional loading reveals that material instability can occur in a diffuse form or be localized in a shear band. Imaging techniques have been used to analyze shear bands in which plastic deformations are fully developed (Desrues et al. 1983; Desrues et al. 1996).

Besides quasi-static deformations, which may be coined as "Coulomb regime", granular materials can be found in at least two dynamic regimes depending on the time scales involved (Goddard 2014): 1) Inertial flows (Bagnold regime), and 2) granular gases. Stresses in dense inertial flows have been known since Bagnold to scale quadratically with the shear rate (Bagnold 1954), but the full scaling was more carefully analyzed only recently by introducing a dimensionless inertial number and applied to different flow geometries (inclined plane, tube, rotating drum, ...) (GDR-MiDi 2004; da Cruz et al. 2005). This scaling indicates that the Coulomb friction angle can be extended to the inertial regime, where energy is dissipated by both inelastic collisions and friction between grains, and increases with inertial number. In the limit of high shear rates or vibration-induced fluidization, long-lasting contacts disappear and the granular material turns into a gas governed by collisions. Granular gases differ from molecular gases in requiring continuous energy input from outside and, hence, they are never in statistical equilibrium (Walton and Braun 1986; McNamara and Young 1992; Goldhirsch and Zanetti 1993; McNamara and Young 1994). Despite their local inhomogeneities induced by inelastic collisions, they can be described by a kinetic theory in which quadratic velocity fluctuations play the role of granular temperature (Jenkins and Richman 1985). The thermodynamic temperature plays, by definition, no role in the dynamics of granular materials. For this reason, the velocity fluctuations are basically controlled by energy input rate. 
Alongside macroscopic testing and modeling approaches, photoelastic experiments of Dantu (Dantu 1957) and careful measurements by Biarez (Biarez 1962) and Oda (Oda 1972) revealed the highly inhomogeneous and anisotropic nature of granular materials at the grain scale under shearing. Computer simulations by the Discrete Element Method (DEM), based on incremental integration of rigid grain displacements and rotations by accounting for frictional contact interactions between grains, provided for the first time direct access to the full dynamics of grains and evolution of granular fabric (Cundall and Strack 1979; Cundall and Strack 1983; Thornton and Randall 1988; Bathurst and Rothenburg 1988; Moreau 1993). It was then believed that by including microstructural information, a macroscopic model of quasi-static behavior can soon be achieved with internal variables fully based on the granular microstructure. However, packing properties induced by steric exclusions between particles and geometrical disorder are complex and rich.

This was actually the point where the mechanics of granular materials joined the physics of amorphous materials such as liquids and glasses in which the packing of hard grains plays a central role (Bernal 1960; Berryman 1986; Pavlovitch et al. 1991; Jullien et al. 1992; Torquato 2010). The concept of Random Close Packing (RCP) was revisited in this context, (O'Hern et al. 2003; Donev et al. 2005; Agnolin and Roux 2007a; Peyneau and Roux 2008a) and that of jamming was introduced (Berryman 1986; Cates et al. 1998; Liu and Nagel 1998) to characterize stability of equilibria in which external forces are balanced by steric impenetrability constraints. Statistical characterizations of granular microstructure have been pursued for 20 years in a multidisciplinary context. Force distributions (Liu et al. 1995; Radjai et al. 1996; Mueth et al. 1998; Radjai et al. 1998), nonaffine particle displacements (Radjai and Roux 2002; Combe et al. 2015), local rearrangements and particle rotations (Kuhn 1999; Bardet 1994; Oda et al. 1997; Kuhn and Bagi 2004; Estrada et al. 2008) and many other features have been carefully analyzed. A rigorous relation between shear stress and fabric anisotropy was introduced by the partition of stress tensor (Bathurst and Rothenburg 1988; Radjai and Richefeu 2009). But the relevant internal variables for the plastic behavior of granular matter remain still open to current research.

Granular matter came vigorously to the focus of physics through a "sandpile" metaphor of self-organized criticality (SOC) (Bak et al. 1987): A sandpile can be built by allowing grains to fall successively into a substrate. As the pile grows, a steady state is eventually reached without tuning any control parameter (self-organized) and with fluctuations (avalanches of grains on the surface 
of the pile) distributed as a power law (criticality), reflecting the absence of intrinsic time and length scales in this state, as in a second-order phase transition. Although a power-law distribution of grain avalanches was not experimentally observed, the SOC brought to the light the highly nonlinear behavior of granular materials and their phase transformations (from solid-like to liquidlike) under moderate energy inputs or stress increments (Jaeger et al. 1990). That is how simple sand was suddenly transformed into a paradigm of complexity, inviting researchers from various fields to develop a fresh look at old real problems.

Most of research in the past thirty years has focussed on model granular materials. The fact that simple spherical particles with frictional contacts produce nearly all complex behaviors of granular materials, extends the status of simple packings as the core model of all granular materials. This is all the more fortunate that the effects of numerous parameters describing real granular materials can be characterized by comparison with this model. Assemblies of rigid, frictionless particles (Roux 2000; Combe and Roux 2000; Wyart et al. 2005; Peyneau and Roux 2008a) have also been studied as extreme case models in which all mechanical response is determined by geometric constraints. Going away from the simplest models, particle size distribution (PSD) is crucial for both spacefilling and shear properties of granular materials. Surprisingly, recent simulations seem to show that the internal friction angle is not dependent on the PSD since force transmission is controlled by the class of largest particles (Voivret et al. 2009).

The effect of particle shape has been more systematically investigated for a few years, and here again the space-filling aspects are counterintuitive and the shear behavior and its relation with the microstructure are complex (Donev et al. 2004; Azéma et al. 2007; Azéma et al. 2009; Torquato 2010; Katagiri et al. 2010; Nguyen et al. 2014; Jaeger 2015). The concept of jamming transition has emerged in physics for unifying the rheology of granular materials with other amorphous materials such as glasses, colloids and foams (Liu and Nagel 1998). Slight variations of temperature close to such a transition lead to the increase of viscosity by orders of magnitudes in a supercooled liquid in the same way as small variations of packing fraction in a granular material control transition from a liquid-like to a solid-like state (Jaeger 2015). This emergence of shear strength is quite rich when particles of strongly nonconvex shape are considered. By monitoring particle shape parameters and assembling method, innovative structures may be designed with applications to material science, architecture and engineering mechanics (Reis et al. 2015). 
Working with objects such as grains is different from dealing with laws governing a continuum in that the grains materialize the degrees of freedom of a packing. It is maybe this essence that makes granular materials so accessible and attractive to many people. Deriving coarse-grained laws arising from the collective behavior of grains is a fundamental research goal. This has been and will be the goal of soil mechanics, which has a long background of engineering practice and deals with granular materials on an everyday basis. The increasing interest in the grain-scale behavior and micro-mechanical approach is not just a luxury. It reflects a real need for enhanced understanding and predictive modeling of soils and powders (Mitchell and Soga 2005). In this endeavor, continuum mechanics provides the framework for a rational analysis but the shift towards multi-scale modeling is unavoidable. The query is how much of the rich information obtained at the micro-scale is relevant to the macro-behavior.

In the following, the authors discuss some major milestones in each of the aspects briefly mentioned above. In practice, these aspects are developed by different communities and their relationships are not always well understood. But it is useful to mention here, even very briefly, relevant contributions for a broad understanding of the field. The first section reviews classical continuum modeling as elaborated, essentially in the soil mechanics community, before roughly 1990. Next, the particle-scale behavior is considered in terms of granular texture, force transmission and particle displacements, followed by a number of bottom-up developments based on particle-level investigations, which benefited, in the three last decades, from a renewed interest in the condensed matter physics laboratories, and from micromechanical approaches. Finally, more recent developments in macroscopic modeling are reviewed. The last section will describe some of the new frontiers and open issues for future research.

\section{CONTINUUM MODELS}

Granular materials can be found in gas-like, liquid-like and solid-like states, but, unlike molecular systems, they are governed by dissipative interactions and steric constraints that make their behavior depend on both pressure level and porosity. Furthermore, as in colloids and foams, their plastic deformations are controlled by substantial evolution of particle arrangements. In this section, the foundations of the continuum models of granular behavior are briefly discussed with pressure, void ratio and anisotropy as basic variables. 


\section{Critical State Theory (CST)}

Granular materials are characterized by both material variability (grain shapes and size distributions, grain surface properties ...) and microstructural variability (organization of grains and their contacts). The incremental stress-strain response of a granular material depends on minute details of its microstructural state, encoding the past deformations and stresses experienced by the material, as shown in Fig. 1. Under complex loading, the microstructure evolves but the memory of the initial state persists and determines the mechanical response. For this reason, the possibility of a unique constitutive framework for the plastic behavior of granular materials with measurable internal variables remained out of reach for a long time. However, a host of mechanical tests on soils in the first half of the $20^{t h}$ century gradually established the fact that a sheared soil tends to a well-defined state in which the memory of the initial state is fully lost.

In this critical state (CS), as put by Casagrande and formulated by Roscoe et al. in 1958, the behavior of granular materials can be described by relationships among shear strength, mean effective stress $p$ and void ratio $e$ (or equivalently packing fraction $\rho=1 /(1+e)$ ) (Casagrande 1936; Roscoe et al. 1958). Using $p=\operatorname{tr}\left(\sigma_{i j}\right) / 3$ and $q=\left(3 s_{i j} s_{i j} / 2\right)^{1 / 2}$, where $\sigma_{i j}$ is the effective stress tensor carried by the granular backbone and $s_{i j}=\sigma_{i j}-p \delta_{i j}$, the CS is generally described by two relationships (Schofield and Wroth 1968; Wood 1990):

$$
\begin{aligned}
\eta & =\frac{q}{p}=M\left(\theta_{L}\right) \\
e & =f\left(p / p_{i}\right)
\end{aligned}
$$

where $f\left(p / p_{i}\right)$ is a function of the effective mean stress $p$ and characteristic stresses $p_{i}$ of the material such as the crushing strength or the cohesive strength, and $M$ characterizes the frictional behavior depending on $\varphi$ and intermediate stress $\sigma_{2}$ via Lode angle $\theta_{L}$. An important observation is that the CS may well be localized inside a sub-volume such as a shear band (Coumoulos 1967). The Critical State Theory (CST) is based on the assumption of homogeneity and isotropy of the material. Objective strains can only be measured in a volume where uniform deformation occurs.

The identification of CS makes it also possible to characterize different states of a granular sample by state variables based on the "distance" from the CS (Been and Jefferies 1985; Jefferies 1993). This is, for example, the case for void ratio $e$, whose evolution largely depends on its 
distance $\Psi=e-e_{c}$, as a state parameter, from the CS line $e_{c}(p)$, dilatant if below the CS line and contractant if above the CS line. The function $f$ in (2) may also depend on the lowest and largest void ratios, $e_{\min }$ and $e_{\max }$, accessible to a granular material (depending on its grain properties). For example, the following fitting form provides a simple function that works reasonably well for sands (Verdugo and Ishihara 1996):

$$
e=e_{\max }-\frac{e_{\max }-e_{\min }}{\log \left(p_{i} / p\right)}
$$

As will be seen below, the state parameter $\Psi$, with its variants, plays a central role in most constitutive models developed for sand. '

\section{Stress-dilatancy relation}

Besides critical states, which reflect self-sustaining microstructures of a granular material under monotonic shearing, energetic considerations were used to derive the first constitutive models of sands and soils. The fact that both stress ratio and void ratio vary with shear strain led Taylor to consider the work done by shear and dilation and formulate a stress-dilatancy relation in 1948 (Taylor 1948). Let $\dot{\varepsilon}_{p}$ and $\dot{\varepsilon}_{q}$ be the volumetric and shear deformation rates in a planar shear flow. The total rate of supplied work is $\dot{W}=q \dot{\varepsilon}_{q}+p \dot{\varepsilon}_{p}$. Hence,

$$
\eta=\frac{q}{p}=-\frac{\dot{\varepsilon}_{p}}{\dot{\varepsilon}_{q}}+\frac{\dot{W}}{p \dot{\varepsilon}_{q}}
$$

For a constant value of $p \dot{\varepsilon}_{q}$ (shear rate and mean stress being imposed) and assuming that $\dot{W}$ is nearly constant, equation (4) suggests a linear relation between $\eta$ and dilatancy defined by $D=-\sin \psi \equiv \dot{\varepsilon}_{p} / \dot{\varepsilon}_{q}$. The dilatancy $D$ vanishes in the CS and, according to (4), $\eta=M=\dot{W} / p \dot{\varepsilon}_{q}$ in the critical state so that

$$
\eta=D+M
$$

The above relation is in good agreement with experiments and simulations only in a limited range of stresses. Alternative versions of this relation did not alter the fundamental conclusion that dilatancy plays a major role in stress-strain behavior of granular materials (Newland and Allely 1957; Rowe 1962; Nova 1982; Bolton 1986). In particular, it controls the shear peak in dense sand and compaction for loose sand. To account for experimental observations, it was modified by Nova 
by introducing a new parameter $N$ such that $\eta=(N-1) D+M_{\theta}$ with the material-dependent parameter $N$ (Nova 1982). The value of the latter is observed to be $\simeq 0.2$ (Jefferies and Been 2006).

However, a more fundamental criticism was introduced by Li and Dafalias (Li and Dafalias 2000). By recalling the basic observation that the same value of $\eta$ may give rise to either contractive or dilatant behavior depending on the current void ratio and/or mean pressure, they remarked that dilatancy can not be a unique function of the stress ratio $\eta$ (unless little change occurs in the internal state) but it should depend on the current state of the material. They showed that major effects of the current void ratio and effective stress can indeed be captured by making depend $D$ on the state parameter $\Psi=e-e_{c}$ in such a way that $D$ vanishes for $\Psi=0$ and $\eta=M$. This requirement is satisfied by the function

$$
D=D_{0}\left(e^{m \Psi}-\frac{\eta}{M}\right)
$$

where $D_{0}$ and $m$ are material parameters. This function suggests that the dilatancy $D$ depends on the difference of the current stress ratio $\eta$ from a reference stress ratio $M e^{m \Psi}$, as in Rowe's stressdilatancy theory but with the difference that this reference stress ratio varies with $\Psi$ instead of being fixed. A similar concept was actually used by Manzari and Dafalias for constitutive modeling (Manzari and Dafalias 1997).

\section{Constitutive models of granular plasticity}

It is important to emphasize that the CST is not a constitutive model but a theoretical expression of robust observational facts. The granular behavior can be described by various elasto-plastic constitutive models of increasing complexity incorporating the CS as a reference state (Manzari and Dafalias 1997; Radjai and Roux 2004; Li et al. 2012; Gao et al. 2014). Cam-Clay model was the first consistent constitutive model based on the CST and Taylor's stress-dilatancy relation, together with the assumptions of associated plasticity and isotropy (Roscoe and Schofield 1963; Schofield and Wroth 1968). In this framework, the stress-dilatancy relation is used to derive a yield surface in which the pre-consolidation pressure plays the role of history parameter. The predictions of this model and its modified version, in both drained and undrained triaxial tests reproduce correctly many observed features of clay behavior under triaxial conditions. The gradual plastification of the clay before stress peak is accounted for by introducing a sub-loading surface evolving isotropically with the state parameter (Hashiguchi 1979; Hashiguchi and Chen 1998). 
Nor-Sand is a model similar to Cam-Clay based on the CST but specialized to sand (Jefferies 1993). The yield surface is derived from Nova's stress-dilatancy relation (Nova 1982), and the pre-consolidation pressure is replaced by the critical state pressure. Nova's stress-dilatancy relation leads to the following yield function:

$$
F=\eta-M_{\theta}\left\{1+(N-1)\left(\frac{p}{p_{i}}\right)^{N /(N-1)}\right\}
$$

where $p_{i}$ is a pressure parameter replacing $p_{c}$ and representing the critical state pressure. To account for plastic hardening, it is further assumed that the stress state lies on a sub-loading surface similar to the above yield surface with varying value of $p_{i}$ until a limit value $p_{i}^{\max }$ is reached. In other words, $p_{i}$ scales the size of the yield surface. The surface evolves as a function of the state parameter $\Psi$. The pressure $p_{i}^{\max }$ is a reference parameter from which the evolution of the $p_{i}$ can be simply modeled by a linear law: $\partial p_{i} / \partial \varepsilon_{p}=H\left(p_{i}^{\max }-p_{i}\right)$, where $H$ is the hardening modulus.

The isotropic nature of the plastic model used in Nor-Sand and similar models, makes them inadequate for modeling cyclic behavior. Volumetric hardening models are adequate for clays, but for granular materials the shear hardening is an essential ingredient. For this reason, and also due to practical calibration problems, later developments in this framework focused mainly on a more general plastic framework with kinematic hardening (Mroz et al. 1978; Manzari and Dafalias 1997).

These concepts are more particularly incorporated in SANISAND, the name for a class of Simple ANIsotropic SAND constitutive models extended from the original two-surface plasticity model developed by Manzari and Dafalias (Manzari and Dafalias 1997; Dafalias and Manzari 2004; Taiebat and Dafalias 2008). This model is based on the CST and bounding surface plasticity, which represents the memory of the material. It uses a narrow open cone-type yield surface obeying rotational (kinematic) and isotropic (volumetric) hardening. The rotational hardening is assumed to reflect the evolution of structural anisotropy. The plastic strain rate is composed of two parts resulting, respectively, from the change of stress ratio (dilatancy) and loading under constant stress ratio. The isotropic hardening depends on the volumetric component of the latter. This model is non-associated and the state parameter $\Psi$ is used to define the peak and dilatancy stress ratios. When calibrated by experiments, it is able to correctly describe the behavior of sand under general conditions, both monotonic and cyclic. It should be noted that the most recent constitutive 
models account for the anisotropy and its evolution by extending the concept of the critical state and introducing state-dependent stress-dilatancy relations (Li et al. 2012; Gao et al. 2014). Such models account naturally for the non-associated feature of flow rule and non-coaxiality between strains and stresses (see section 5).

The above models assume the concept of simple materials (Noll 1955), which implies that the material behavior can be fully characterized under homogeneous strains. Shear banding, however, seems to counter this assumption. One of the early constitutive models fully based on slip planes was the dilatant double shearing model of Mehrabadi and Cowin (Mehrabadi and Cowin 1978). In this model, it is assumed that the deformation of a granular material is composed of two dilatant shear deformations along the stress characteristics, which are interpreted as slip lines. This model in its later developments was enriched by incorporating elastic deformation, plastic work hardening and, more recently, the fabric tensor (Nemat-Nasser et al. 1981; Zhu et al. 2006). The shear rates along the two slip systems are related to the density of contacts along them. While several internal variables introduced in the model are phenomenological, the model can describe monotonic and cyclic responses of granular materials, the evolution of void ratio and the evolution of fabric tensor with the stress tensor (see section 3).

It is useful to briefly mention also here the framework of hypoplasticity, which was developed with the strong idea of finding the most general and objective expression of the stress rate $\dot{\sigma}$ as a function of the current stress state $\sigma$ and velocity gradient tensor $D$ (Kolymbas 1991; Kolymbas et al. 1995; E. 1996; Gudehus 1996; Lanier et al. 2004). The application of representation theorems of isotropic functions leads to a generically nonlinear dependence of the material behavior on the direction of strain rate. The incremental nonlinearity being intrinsic to the evolution equation, there is no need to explicitly distinguish between loading and unloading paths. This framework postulates no yield function and flow rule, and the usual partition of the strain-rate tensor into elastic and plastic parts disappears. However, those concepts can be obtained as predictions of the model for different forms and values of model parameters (Wu and Niemunis 1996).

Advanced hypoplastic models account for void ratio as scalar state variable and pressure level, as well as the critical state (Huang et al. 2008; Masin 2012; Fuentes et al. 2012). The tensorial fabric state can also be incorporated in the model at the price of extending the framework and number of material parameters (Wu 1998). The latest developments tend to show that almost all elasto-plastic 
models can be equally formulated in the hypoplastic framework (Huang et al. 2006). A unification was actually achieved by Einav, who considered the class of hypoplastic models that are compatible with thermodynamics and showed that elasto-plasticity is a singular limit of hypoplasticity (Einav 2012).

Another general framework applied to granular materials is that of micropolar theories, which, by introducing a length scale into their continuum description, offer the possibility of accounting for size effects, e.g. in shear banding. This length may be interpreted as the scale below which particle rotations can significantly differ from those deduced from the rotational of the displacement field. At a larger scale the difference vanishes and thus, upon coarse-graining, the standard theory is recovered (Tejchman and $\mathrm{Wu} 1993)$. These models attribute micro-rotations and couple stresses to material points, extending thus the number of constitutive relations for elastic and plastic behavior. In association with hypoplasticity, they can lead to realistic results in large deformations with shear banding (Grammenoudis and Tsakmakis 2005).

\section{Elastic behavior}

An elastic or quasi-elastic response is observed in granular materials when small stress increments are added to a pre-stressed equilibrium configuration by static or dynamic (resonance, sound propagation) experiments. The linear response to such increments is then, as a good approximation, reversible, and associated to some quadratic elastic energy, the initial prestressed configuration defining the reference state, which is at the origin of elastic displacements and strains. An elastic model applies, for sands stabilized under confining stresses between $10 \mathrm{kPa}$ and a few MPa, to strain intervals not exceeding some limit of order $10^{-6}$ or $10^{-5}$ (Hicher 1996; Shibuya et al. 1992; Kuwano and Jardine 2002; Geoffroy et al. 2003). For larger strains, the dissipation is no longer negligible in stress-strain cycles.

The accuracy of strain measurements in soil mechanics has made significant progress over the last decades, and measurements in the very small strain, quasi-elastic regime, about differently prestressed states are now feasible. Static measurements of elastic moduli agree with dynamic measurements deduced from resonance wave frequencies or propagating wave speeds (Thomann and Hryciw 1990; Shibuya et al. 1992; Geoffroy et al. 2003). The elastic moduli should not be confused with the slopes of stress-strain curves on the strain scale (say, of the order of $1 \%$ ) corre- 
sponding to the mobilization of internal friction. Those slopes are considerably smaller than true elastic moduli, by more than an order of magnitude, and do not correspond to an elastic response. Simplified elastic-plastic models (Vermeer 1998) used in engineering applications, in which the material is linear elastic until the Mohr-Coulomb criterion for plasticity is reached, corresponding to full internal friction mobilization, should not be misinterpreted: the elastic part of the material behavior in such models is essentially a computationally motivated simplifying assumption.

\section{PARTICLE-SCALE BEHAVIOR}

Granular materials are disordered at the micro-scale with strong fluctuations of particle environments from one particle to another. Within the rigid-particle approximation, the local behavior can be described by vectorial quantities (branch vectors, contact forces), which underly macroscopic tensorial variables (stress, strain, fabric). In this section, the main features of granular texture, force transmission and particle displacements are discussed.

\section{Granular texture}

In a fundamental approach, the state parameters are of geometrical nature. Such descriptors of the texture are generally known as fabric parameters. The level of description depends on the choice of these parameters, which should naturally comply with both the accuracy and tractability of the formulation and which can be scalar or tensorial parameters or functions. Two constraints make the plastic behavior of granular materials depend on nontrivial aspects of the microstructure: 1) the steric hindrances among neighboring particles, which constrain the accessible geometrical states, and 2) the condition of mechanical equilibrium, which controls to some extent the range of admissible particle configurations (Roux and Radjai 2001; Troadec et al. 2002; Radjai 2009).

Figure 2(a) displays a representation of the contact geometry between two neighboring particles. The relevant geometrical variables are the contact vector $\vec{r}$ joining the particle center to the contact point, the branch vector $\vec{\ell}$ joining the centers of two contacting particles and the contact orientation vector (contact normal) $\vec{n}^{\prime}$ defined as the unit vector normal to the particle boundary at the contact

zone $\alpha$. The reaction forces $\vec{f}$ and $-\vec{f}$ acting on the two particles at their contact zone have a unique application point that may be considered as their contact point. The local frame is composed of the radial unit vector $\vec{n}$ and one orthogonal unit vector $\vec{t}$ in an ortho-radial plane (orthogonal to the contact vector). In $2 \mathrm{D}$, the local frame is uniquely defined by a single tangent unit vector $\vec{t}$. 
One needs a statistical description due to granular disorder, with the basic feature that the local vectors vary discontinuously from one contact to another. The local environments fluctuate in space both in the number $k$ of the contacts of each particle (topological disorder) and in their angular positions $\vec{r}^{\alpha}$ (metric disorder). For the formulation of the local constraints only the first contact neighbors of a particle are sufficient. Two functions are required to describe this first shell environment (Roux and Radjai 2001; Troadec et al. 2002):

1. $P_{c}(k)$ : Connectivity function defined as the proportion of particles with exactly $k$ contacts (first shells with $k$ members).

2. $P_{k k r f}\left(\vec{r}^{1}, \ldots, \vec{r}^{k}, \vec{f}^{1}, \ldots, \vec{f}^{k}\right):$ Multicontact probability density function of $k$ contact forces $\vec{f}^{\alpha}$ and $k$ angular positions $\vec{r}^{\alpha}$ for a shell of $k$ particles.

The average connectivity of the contact network is the coordination number $z=\sum_{k=1}^{\infty} k P_{c}(k)$. Integration of $P_{k k r f}$ over all angular positions yields the multicontact force pdf's $P_{k k f}\left(\vec{f}^{1}, \ldots, \vec{f}^{k}\right)=$ $\int_{\mathcal{A}_{k r}} P_{k k r f} d\left\{\vec{r}^{\alpha}\right\}$, where $\mathcal{A}_{k r}$ is the accessible domain of angular positions. In the same way, the multicontact pdf of angular positions is $P_{k k r}\left(\vec{r}^{1}, \ldots, \vec{r}^{k}\right)=\int_{\mathcal{A}_{k f}} P_{k k r f} d\left\{\vec{f}^{\alpha}\right\}$, where $\mathcal{A}_{k f}$ is the integration domain. In the particle shells, the steric constrains manifest themselves as angular exclusions. Two particles belonging to a shell cannot approach one another below a minimum angular interval $\delta \theta_{m i n}$; see Fig. 2(b). In other words, the multicontact probability density $P_{k k r}$ vanishes if the angular exclusions are violated.

These multicontact probability density functions are too rich to be accessed experimentally or tackled theoretically. The information that they contain can be reduced in three steps. In the first step, one extracts the 1-contact distributions for the shells of $k$ contacts by integration over all contacts except one. In the second step, the 1-contact distributions are averaged over the shells by weighting them by $P_{c}$. The third step consists in extracting the angular behavior. In particular, the probability density function $P(\vec{n})$ of contact normals is often used to represent the structural anisotropy. Its second moment $\mathbf{F}=\langle\vec{n} \otimes \vec{n}\rangle \equiv \int_{\Omega} \vec{n} \otimes \vec{n} P(\vec{n}) d \vec{n} / 2 \pi$, where $\Omega$ denotes the angular domain, defines the fabric tensor $\mathbf{F}$ with $\operatorname{tr}(\mathbf{F})=1$. The lowest-order anisotropies of the contact network are given by $a_{c}^{i j}=2\left(F_{i}-F_{j}\right)$, where $F_{1}, F_{2}$ and $F_{3}$ are the principal values of $\mathbf{F}$ (Oda et al. 1980; Satake 1982; Rothenburg and Bathurst 1989; Cambou 1993; Radjai et al. 1998; Kuhn 1999; Ouadfel and Rothenburg 2001). Higher-order moments and anisotropies can also be extracted from 
$P(\vec{n})$.

Obviously, the fabric anisotropy can equivalently be defined from other characteristic orientations such as those of branch vectors (joining particles centers) or voids, which generally have elongated shapes (Satake 1982; Kuhn 1999). The choice should naturally be guided by the modeling approach and the variables of interest. The yield function is more closely related to contact anisotropy or branch-vector anisotropy since the condition of force balance is enforced at the level of each particle with forces exerted by neighboring particles whereas the flow rule reflects the evolution of void volumes for which the compatibility condition of particle displacements can be formulated, and hence the void anisotropy and valence number (average number of particles in a loop of particles) seem to be essential; see Fig. 2(c). The connection between these two anisotropies is an important ingredient for relating local strains and stresses in a micromechanical approach (Roux and Radjai 2001). A related aspect is the scale at which the fabric is defined. Particle-scale fabrics are fluctuating variables both in space and in time. They are well-defined only at a mesoscopic scale. In this sense, the averaged fabric tensors are macroscopic objects and their use in a macroscopic model assumes that the Representative Volume Element (RVE) exists not only for stresses and strains but also for the fabric tensors. In contrast, a micromechanical approach should be based on particle-scale fabric variables, which incorporate the constraints of force balance and kinematic compatibility (Troadec et al. 2002).

\section{Stress transmission}

The distribution of contact forces in model granular materials has been extensively studied initially by experiments and later amplified by Discrete Element Method (DEM) simulations. Geometrical exclusions and disorder in granular materials lead to a highly inhomogeneous distribution of forces (Dantu 1957; Drescher and de Josselin de Jong 1972; Liu et al. 1995; Radjai et al. 1996; Thornton 1997; Mueth et al. 1998; Radjai et al. 1999; Majmudar and Behringer 2005; Miller et al. 1996). Filamentary patterns of stresses observed on photoelastic images are induced by strong contact forces and are known as force chains. The forces were more accurately measured by using carbon paper to record normal force prints at the boundaries of a bead packing (Mueth et al. 1998). They were found to have a nearly uniform probability density function (pdf) in the range of weak forces followed by an exponential falloff of strong forces. Similar force distributions were found by 
means of numerical simulations (Radjai et al. 1996; Thornton 1997; Radjai and Wolf 1998; Radjai et al. 1999; Antony 2001).

All further investigations of force distributions have shown that the exponential distribution of strong forces is a robust feature of granular media. In contrast, the weak forces appear to be sensitive to the packing state resulting from the deformation history (Antony 2001; O'Hern et al. 2001; Snoeijer et al. 2004). In an isotropic packing state, the distribution shows a relatively small peak below the mean force, and the probability density of small forces does not fall to zero (Metzger 2004a; Metzger 2004b). The peak disappears in a sheared packing and the distribution of weak forces turns to a nearly decreasing power law (Radjai et al. 1999; Antony 2001; Azéma and Radjai 2014). Fig. 3(a) shows a map of contact forces in a strongly polydisperse packing. This is what generally is observed in frictional packings composed of aspherical grains or broad size distributions (Voivret et al. 2009; Azéma et al. 2007; Azéma et al. 2009; Saint-Cyr et al. 2011; Azema et al. 2013; Nguyen et al. 2014). Increasing the confining stress of elastic grains leads to considerable deformation of the grains and increase of the coordination number beyond its rigid limit value, while the force distribution tends to assume a narrower nearly Gaussian shape (Makse et al. 2000; Agnolin and Roux 2007b).

The $q$-model was the first statistical model of force distributions (Liu et al. 1995; Coppersmith et al. 1996). The forces are assumed to be scalar quantities that "propagate" from site to site (particles) along the links (contacts) of a regular network. The total incoming force from the particles of a layer to each site is redistributed to the particles of the next layer according to a random process. This model predicts that the force pdf converges to a purely exponential function $P(f)=\beta e^{-\beta f}$ with the exponent $\beta$ depending on the number of supporting contacts per grain. A statistical approach developed by Metzger et al. provides a correct estimate of the force pdf by analyzing the local constraints and accounting for the density of states in a first-shell approximation (one grain with its contact neighbors) (Metzger 2004b). Another simple analytical model based on the assumption that a force of a given value can only be generated from the population of higher forces but requires weaker forces for its equilibrium, leads to an expression with a single free parameter that fits well the force distributions (Radjai 2015).

Detailed analysis of sheared dry granular materials provides evidence for the bimodal organization of the force network in well-defined "weak" and "strong" networks, with the strong network 
contributing almost exclusively to the shear strength, and weak forces acting mainly to prop strong force chains (Radjai et al. 1998; Radjai et al. 1999). The supportive effect of weak forces is reflected in the anisotropy of weak contacts with their privileged direction that is perpendicular to that of strong contacts. In the case of polygonal grains in $2 \mathrm{D}$, this supportive action appears in the anisotropy of the weak forces rather than weak contacts (Azéma et al. 2007).

In the absence of stress gradients (macroscopically homogeneous shear), the forces below the mean are in the weak network, which contains nearly $60 \%$ of contacts, which sustain $25 \%$ of the average stress. Nearly all sliding contacts during shear belong to this network, i.e. plastic dissipation occurs almost fully in this network. But since the partial shear stress through this network is nearly zero, from a mechanical viewpoint it is a liquid-like phase whereas the strong network behaves as a solid skeleton for the medium. This bimodal feature suggests that granular materials can be modeled as two-phase media with two stress tensors (Zhu et al. 2006).

\section{Granular kinematics}

Kinematic fields (particle translations and rotations) at the particle scale in sheared granular materials have complex patterns that have been much less investigated than contact force distributions in exception to shear localization, which has for a long time been associated with failure at peak stress ratio. DEM simulations show that even at early stages of shear deformation, wellorganized micro-bands of intense shearing occur despite overall homogeneous boundary conditions (Kuhn 1999; Lesniewska and Wood 2009). These micro-bands evolve rapidly in space and time, and the shear bands at larger strains seem to arise as a result of their coalescence.

Grain rotations and rolling contacts play a crucial role in the local kinematics of granular materials. Shear zones are generally marked by intense particle rotations (Oda et al. 1982; Kuhn and Bagi 2004). Since rolling contacts dissipate much less energy than sliding contacts, grain motions in quasi-static shear occur mostly by rolling. Sliding contacts are actually a consequence of the frustration of particle rotations in the sense that all contacts within a loop of contiguous particles cannot be simultaneously in rolling state. The basic structure of a loop of three grains illustrates well this property (Tordesillas 2007). For this reason, it has been argued that such mesoscopic structures evolve and their statistics is correlated with plastic hardening and softening of granular materials. In general, long-range correlations, as those reflected in the structure of force 
chains, indicate that single-contact models cannot fully capture the local behavior. A correlation length of the order of 10 grain diameters is observed in forces (Staron et al. 2005). As an internal length scale, it can be related to the thickness of shear bands.

Another important feature of particle velocity fields is that, as a result of steric exclusions, the particle velocities $\left\{\vec{r}^{i}\right\}$ have a non-affine fluctuating component $\left\{\vec{s}^{i}\right\}$ of zero mean with respect to the background shear flow, as displayed in Fig. 3(b) (Radjai and Roux 2002; Peters and Walizer 2013; Combe et al. 2015). These fluctuating velocities have a scaling behavior which is very similar to those of fluid turbulence and were therefore coined by "granulence" by Radjai and Roux (Radjai and Roux 2002). In particular, the velocity probability density functions undergo a transition from stretched exponential to Gaussian as the time resolution is increased, and the spatial power spectrum of the velocity field obeys a power law, reflecting long-range correlations and the selfaffine nature of the fluctuations. These observations contradict somehow the conventional approach which disregards kinematic fluctuations in macroscopic modeling of plastic flow in granular media. The long-range correlations of velocity fluctuations may be at the origin of the observed dependence of shear stress on the higher-order gradients of shear strain, implying that granular materials are not "simple" materials in the sense of Noll (Kuhn 2005; Noll 1958).

A key aspect of granular kinematics is its discontinuous evolution. The contacts between particles have a short lifetime and new contacts are constantly formed as the particles move. The overall picture is that more contacts are gained along the directions of contraction and lost along the directions of extension (Rothenburg and Bathurst 1989). A detailed balance equation can be written for the evolution of contacts along different directions. Only in the critical state the rates of gain and loss are equal (Radjai et al. 2004; Radjai et al. 2012). However, this is only an average picture and the effects of the chaotic fluctuating velocity field are not yet well understood (Pouya and Wan 2016). They may well behave as a noise or control the fabric evolution under complex loading conditions. When the direction of shear is reversed, for example, there is a clear asymmetry between the gain and loss processes, which leads to a decrease of the coordination number whereas the void ratio decreases at the same time (Radjai and Roux 2004). Such effects control the nonlinear behavior of granular materials under complex loading paths. 


\section{BOTTOM-UP APPROACH}

Beyond experimental validation, macroscopic models of granular behavior must provide a clear physical basis for their internal variables (or state parameters) at the grain scale. Considerations on grain-level mechanics and statistics of contact networks and their rearrangements, on the one hand, and ideas from condensed-matter physics and soft matter rheology, on the other hand, triggered or inspired different methods for bottom-up (micro-macro) modeling of granular materials that are discussed in this section.

\section{Numerical and multiscale modeling}

The bottom-up modeling refers to an approach fully or partially based on the particle interactions and granular texture. This implies upscaling through at least five scales: contact, particle, assembly, representative volume element (RVE) and structure, as schematized in Fig. 4. The upscaling from the contact scale is mediated by particles (where equilibrium conditions are defined), and their assembly in mesoscopic microstructures (where compatibility conditions are defined).

Besides extraordinary progress made in measurement techniques, computerized testing and imaging devices such as micro-tomography, the DEM (Discrete Element Method) allows accurate simulations of stiff particles for complex loading and significant cumulative strains. This has made it possible to characterize granular texture, force transmission and particle motions, and to address long-lasting issues such as the role of fluctuations, partial stresses carried by a specific class of contacts or particles ..., which are clearly much more complex than naive pictures of local behavior used sometimes in earlier developments. Such details are, however, not straightforward to fit into continuum modeling approaches. Hence, the issue is to identify the lowest-order geometrical parameters that should be incorporated in a macroscopic model and understand the extent that mechanical behavior depends on higher-order parameters. This approach is not straightforward and has many drawbacks compared to advanced constitutive models, but will lead in the long run to predictive models.

However, the DEM is not yet computationally efficient for solving boundary-value problems at large scales with large number of particles. For this reason, the DEM can be used as a means to access the meso-scale (scale of an assembly of grains) information, which can then be upscaled to the continuum level using the Finite Element Method (FEM) or averaging techniques (Kaneko 
et al. 2003; Andrade and Tu 2009; Nitka et al. 2011; Andrade et al. 2011; Guo and Zhao 2016; Liu et al. 2016). Most of the recent work in the physics community addresses the first stage, i.e. the description of meso-scale structures from the grain-scale, whereas many micromechanical models are concerned with the second stage in which the meso-scale is represented as a continuous distribution of contact planes.

The multiscale approach is often mentioned as an alternative to purely numerical DEM or micromechanical approaches. The FEM is employed to solve a boundary value problem while using the DEM to derive the required nonlinear material responses at each FEM Gauss integration point. Hence, this approach requires no constitutive model as in the conventional FEM simulations. The accuracy of the model depends on the number of Gauss points (FEM discretization) and the number of particles associated with each point (DEM discretization). This approach should therefore be considered as a hierarchical coupling approach and an integrated tool for solving boundary-value problems. But it should not elude a theoretical micromechanical approach with the goal of basing macrosopic models on internal variables pertaining to the granular texture for a better understanding the origins of the complex phenomenology of granular materials.

\section{Stress and strain tensors}

The discrete nature of granular materials makes them different from composites or other inhomogeneous materials usually considered in micromechanics. In particular, the macroscopic tensorial variables such as stress and strain must be constructed from statistical averages of vectorial variables such as contact forces and relative displacements of grains over representative volume elements (RVE). As pointed out by He, the definition of macroscopic stress and strain tensors should be based on the boundary data of a RVE since they should satisfy the equilibrium and compatibility equations, respectively (He 2014). For granular materials, the expression of the stress tensor as a function of contact forces and branch vectors is classical since equilibrium conditions are well defined (Love 1929) whereas compatibility equations are less well formulated.

The approach recently introduced by He consists in considering grain centers and taking the domain generated and partitioned by the Delaunay tessellation as the domain of a representative volume element filled with a continuum medium (He 2014). Following a similar work by Kruyt and Rothenburg in 2D (Kruyt and Rothenburg 1996), the 3D compatibility equations for the 
grain center displacements is thus established and an explicite micromechanical expression for the macroscopic strain rate is derived. The expressions of stress and strain rate tensors are

$$
\begin{aligned}
\sigma_{i j} & =n_{c}\left\langle f_{i} \ell_{j}\right\rangle_{c} \\
\dot{\varepsilon}_{i j} & =n_{p}\left\langle\delta \dot{u}_{i} a_{j}+\delta \dot{u}_{j} a_{i}\right\rangle_{p} / 2 V
\end{aligned}
$$

where $n_{c}$ is the number density of contacts, $n_{p}$ is the number density of neighboring grain pairs (pairs of grains in contact or separated by a small gap), $\ell_{j}$ is the $j$-component of the branch vector joining grain centers, $f_{i}$ is the $i$-component of contact force, $V$ is the total volume, $\delta \dot{u}_{i}$ is the $i$-component of the relative velocity between two neighboring grains, and $a_{j}$ is the $j$-component of the area vector perpendicular to the Delaunay plane separating the the two grains. The first averaging runs over all contacts $c$ whereas the second one runs over all adjacent pairs of grains.

\section{Granular elasticity}

The elasticity of a granular packing is a direct consequence of the elasticity of particles, as expressed in contact laws (Johnson 1985). Elastic moduli of granular packings are primarily sensitive to the stress level, via the average contact stiffness, which is proportional to $P^{1 / 3}(z \phi)^{-1 / 3}$ under pressure $P$, where $\phi$ is the solid fraction and $z$ is coordination number, if contacts are Hertzian (factor $z \Phi$, expressing the contact density, appears here because the average contact force scales as $P /(z \Phi)$ (Agnolin and Roux 2007a)). Assuming contacts through angular asperities (Johnson 1985), local stiffnesses should rather scale as $P^{1 / 2}(z \phi)^{-1 / 2}$. Such power law stress dependences are experimentally or numerically observed, with, most often, an exponent slightly larger than $1 / 3$ for spherical particles (Kuwano and Jardine 2002; Agnolin and Roux 2007c), or than 1/2 for angular sand grains (Hicher 1996). This difference between predicted and measured exponent values might partly be attributed to the change of contact numbers under varying stresses (Goddard 1990b), although increases of coordination numbers under growing stresses is mostly influential (Makse et al. 1999; Agnolin and Roux 2007c) under rather large confining stress levels (in the MPa range).

Recent numerical studies show that the departure from theoretical exponents, under lower pressure, is most often due to the singular properties of poorly connected networks (Wyart 2006; Somfai et al. 2005), in which the small degree of force indeterminacy entails (Wyart et al. 2005) a large excess of "soft modes", associated to anomalously low eigenvalues of the contact network stiffness 
matrix, in comparison to those of a homogeneous elastic continuum. In the limit of vanishing force indeterminacy, which is indeed approached for small stresses with frictionless objects (Roux 2000; Silbert et al. 2002), the moduli tend to scale with the degree of force indeterminacy per unit volume (Wyart 2006; Agnolin and Roux 2007c; Somfai et al. 2007), whence a proportionality to the difference between the coordination number of the force-carrying network and its minimum (isostatic) value: to $z-6$ for frictionless spheres, or to $z-4$ for frictional ones. The resulting anomalously low moduli increase faster with pressure, as they are sensitive to small increases of coordination numbers. This is apparent in Fig. 5, showing an increase of shear moduli with pressure, for poorly coordinated sphere packings, with an exponent notably larger than the theoretical value $1 / 3$. Such effects, in isotropic systems, do not influence the bulk modulus, for which simple predictions based on the Voigt assumption of affine elastic displacement fields provide reasonable approximations (Somfai et al. 2007; Agnolin and Roux 2007c). For anisotropic stress states (Peyneau and Roux 2008b), the non-singular modulus is the one associated with stress increments proportional to the pre-existing stress values.

Figure 5 also illustrates the greater sensitivity of moduli to coordination number $z$, than to solid fraction $\Phi$ (Agnolin and Roux 2007c; Magnanimo et al. 2008). $z$ varies, between different preparation procedures, independently of $\Phi$ for dense systems (Agnolin and Roux 2007a; Magnanimo et al. 2008). Of course, elastic moduli also reflect the anisotropy of the reference, prestressed state, both due to stresses and to internal fabric. Anisotropic elastic properties have been probed in experiments for some time (Hoque and Tatsuoka 1998; Kuwano and Jardine 2002; Duttine et al. 2007) and are currently being explored by numerical simulations (La Ragione and Magnanimo 2012a; La Ragione and Magnanimo 2012b).

The elastic behavior, albeit restricted to very small strain intervals, is an essential ingredient in the discussion of incremental elastoplastic properties, the detailed form of which is important for instability criteria. Elastic moduli also indirectly provide access, in a nondestructive way, to geometrical data on the contact network, such as coordination number and fabric anisotropy.

\section{Granular plasticity}

Most micromechanical models are based on a relationship between force and relative displacement vectors on contact planes or mobilized planes, and a dependence of the parameters on the 
distribution of contact planes (Christoffersen et al. 1981; Chang and Hicher 2005; Chang et al. 2009; Chang et al. 2011). Such models are conceptually simple but capture the main features of the stress-strain behavior in monotonic shearing. An important element of such models is the relation between local and global strain or stress variables. A rational assumption is that the forces on each contact plane are equal to the components of the stress tensor. This is obviously not an exact assumption since the contact forces have a highly inhomogeneous distribution. Another possible assumption is a kinematically constrained microstructure in which the local displacements are components of the macroscopic strain tensor. This is equivalent to the assumption that the velocity field has no non-affine components, which is wrong and leads to over-estimated predictions for elastic moduli, for example (Kruyt and Rothenburg 2002a). Another constraint used in micromechanical approach is the equality between energy dissipation rate $\sigma_{i j} \dot{\varepsilon}_{i j}$ and frictional dissipation at all sliding contacts (Cambou 1993). This is a strong assumption since only a small proportion of contacts are critical and the friction forces have a broad distribution. Furthermore, careful numerical simulations indicate that nearly $25 \%$ of energy dissipation in the quasi-static limit is due to inelastic collisions between grains (Radjai and Roux 2004). This energy is dissipated by micro-instabilities during shear and their signature on the critical-state shear stress can be observed in the form of fluctuations.

The current challenge in micromechanics of granular materials is to account for fabric anisotropy required for understanding and modeling complex loading paths (Wan and Guo 2004; Dafalias and Manzari 2004; Li and Dafalias 2015; Radjai 2009; Sun and Sundaresan 2011; Radjai et al. 2012; Chang and Bennett 2015). Because of the problems arising from a proper definition of grain-scale kinematics from the boundary or far-field displacements via a localization tensor, there is presently no general expression for the evolution of the coordination number, fabric tensor and dilatancy. For this reason, most models consider only the effects of geometrical anisotropy by introducing, for example, different elastic moduli for loading and unloading. It should be noted that in a constitutive model developed on the basis of monotonic loading behavior or involving no tensorial internal variable, the response upon unloading is elastic. In practice, however, a granular material is "fragile" in the sense that stress increments in directions opposite to the shear directions cannot be supported without plastic deformation (Cates et al. 1998). This leads to long plastic transients when the direction of shearing is changed before the critical state is reached in the new direction 
(Radjai and Roux 2004). If shear reversal is repeated at small strains, the system is attracted to a new state that has not yet been investigated from a micromechanical viewpoint (Alonso-Marroquin and Herrmann 2002). This state is a consequence of subtle memory effects that pull the packing towards a high density but low coordination number.

\section{Origins of internal friction}

Frictional behavior is the most basic and common feature of the plasticity of granular materials. In contrast to dry friction between two solid bodies, it is a bulk property. It has also been described as friction between two "blocks" of a granular materials separated by a shear band. The localization of shear deformation along a thin interface inside a dense granular material or with a structure is an interesting analogy with dry friction between solid bodies but it masks the general properties of granular friction and those of loose granular materials and steady flows, which cannot simply be described by shear bands.

Granular friction reflects the collective motions of a large number of grains and it has a tensorial nature. In particular, it is not a simple function of friction between grains. The internal (or global) friction coefficient $\mu^{*}$ in steady flow (or in the critical state of soil mechanics) has a non-vanishing value even for frictionless grains (Peyneau and Roux 2008b). The energy dissipation in this limit results from inelastic collisions between grains and, since the collision velocity is proportional to the mean effective stress $p$, it increases proportionally to $p$, as required by a Coulomb-like friction. Furthermore, as the grain-grain friction coefficient $\mu_{s}$ increases, $\mu^{*}$ increases but it levels off for $\mu_{s} \simeq 0.4$ (Taboada et al. 2006). Above this value, $\mu^{*}$ is independent of $\mu_{s}$. The expression (8) of stress tensor makes it possible to calculate from DEM simulations the contribution of friction forces to the total shear stress and hence internal friction angle $\mu^{*}=\tan \left(\sigma_{t} / \sigma_{n}\right)$ along a granular shear flow by considering only the tangential components $f_{t}$ of forces. Quite unexpectedly, this contribution is quite small (below 10\%) (Thornton and Randall 1988).

Another quantity that is expected to correlate with internal friction coefficient is the proportion of sliding contacts or critical contacts, i.e. contacts where friction is fully mobilized $f_{t} / f_{n}=\mu_{s}$. For spherical grains, in the quasi-static state the proportion of sliding contacts is below 10\%. In a granular pile gradually tilted from horizontal to its maximum angle of stability, this proportion increases exponentially towards its maximum value (Staron et al. 2002). The sliding points inside a 
packing can be described as dislocations that carry plastic deformations. Their percolation across a packing eventually leads to failure. This viewpoint is interesting as it hints at avalanche precursors. But it does not provide a clear understanding of the properties of granular friction.

The above observations indicate that the bulk friction of granular materials is not a direct consequence of frictional contacts between grains but rather a structural effect. This was clearly established by a new partition of the stress tensor introduced by Rothenburg and Bathurst (Bathurst and Rothenburg 1988). The expression of stress tensor can be evaluated as an integral from the probability density function $P(\vec{f}, \vec{\ell})$ of branch vectors $\vec{\ell}$ (vectors joining grain centers) and contact forces $\vec{f}$. On needs also to introduce the angular distributions of contact normals $P(\vec{n})$ and force averages $f_{n}(\vec{n})$ and $f_{t}(\vec{n})$, where $\vec{n}$ is the orientation of the branch vectors. In a sheared granular material, these functions can be approximated by their truncated Fourier expansions in 2D (or expansions in spherical harmonics in 3D):

$$
\begin{aligned}
P(\theta) & \simeq \frac{1}{\pi}\left\{1+a_{c} \cos 2\left(\theta-\theta_{c}\right)\right\} \\
\left\langle f_{n}\right\rangle(\theta) & \simeq\langle f\rangle\left\{1+a_{n} \cos 2\left(\theta-\theta_{f}\right)\right\} \\
\left\langle f_{t}\right\rangle(\theta) & \left.\simeq\langle f\rangle a_{t} \sin 2\left(\theta-\theta_{f}\right)\right\}
\end{aligned}
$$

where $\langle f\rangle$ is the average contact force, $\theta_{c}$ is the preferred direction of contacts and $\theta_{f}$ is the preferred direction of forces. The above expressions together with the integral expression of the stress tensor yield two relations (here in 2D) (Bathurst and Rothenburg 1988):

$$
\begin{aligned}
p & =n_{c}\langle f\rangle\langle\ell\rangle \\
\frac{\sigma_{1}-\sigma_{3}}{\sigma_{1}+\sigma_{3}} & =\sin \varphi=\frac{1}{2}\left(a_{c}+a_{n}+a_{t}\right)
\end{aligned}
$$

where $p$ is the mean effective pressure, $\langle\ell\rangle$ is the average branch vector length, $\varphi$ is the internal friction angle, $a_{c}$ is contact orientation anisotropy (fabric anisotropy), $a_{n}$ is normal force anisotropy and $a_{t}$ is tangential force anisotropy. The above expressions are obtained by assuming that the principal major direction $\theta_{\sigma}$ of the stress tensor is the same as $\theta_{c}$ and $\theta_{f}$. This is a reasonable assumption since all directions tend to coincide during a monotonic shear deformation.

Equation (13) is similar to the perfect gas law with contacts playing the role of molecules 
and $\langle f\rangle\langle\ell\rangle$ replacing $k_{B} T$, where $k_{B}$ is the Boltzmann constant and $T$ is absolute temperature. Equation (14) is an explicite expression of the internal friction angle as a function of fabric and force anisotropies. These two equations are in excellent agreement with simulations in monotonic shear deformation. For unmonotonic deformations, the coaxiality of the branch vectors and forces is not verified and hence it is necessary to account for the phase factors (Radjai and Richefeu 2009):

$$
\sin \varphi \simeq \frac{1}{2}\left\{\left(a_{c}+a_{\ell}\right) \cos 2\left(\theta_{\sigma}-\theta_{c}\right)+\left(a_{n}+a_{t}\right) \cos 2\left(\theta_{\sigma}-\theta_{f}\right)\right\}
$$

where the anisotropy $a_{\ell}$ of the branch vector length is also added and described by a truncated Fourier expansion. This expression is nicely verified in simulations both in cohesive and noncohesive materials, and under unmonotonic conditions. This indicates that the internal friction coefficient has three distinct origins: 1) fabric anisotropy $\left.a_{c}+a_{\ell}, 2\right)$ normal force chains captured by $a_{n}$ and 3) friction mobilization quantified by $a_{t}$. The relation between $a_{t}$ and friction mobilization is evident by noting that, according to equation (12), the largest value of the ratio $\left\langle f_{t}\right\rangle / f$ occurs along the direction $\theta=\theta_{f}+\pi / 4$. Equation (15) clearly shows that friction mobilization is not the only factor giving rise to the internal friction but that the contributions of force chains and contact network anisotropy can even be more important.

The respective weights of the above anisotropies vary with particle size distribution (PSD) and grain shapes. For example, in a packing of spherical grains the fabric anisotropy is above the normal force anisotropy whereas in a packing of polyhedral grains the normal force anisotropy is higher than fabric anisotropy (Azéma et al. 2009). This reflects the fact that force chains are reinforced by face-face contacts between polyhedral grains. The fabric anisotropy $a_{c}$ declines as PSD becomes broader but branch vector length anisotropy $a_{\ell}$ increases (Voivret et al. 2009). The force anisotropies remain unchanged since force chains are captured by the class of largest grains. As a result, the internal friction angle remains nearly independent of PSD.

It is often assumed that the shear strength of granular materials increases with their compactness measured in terms of their void ratio $e$ and/or coordination number $z$. However, the compactness does not explicitly appear in equation (15). Hence, the effect of compactness on the shear strength is mediated by the fabric and force anisotropies. The steric exclusions in the close environments of the grains imply that the maximum value of fabric anisotropy decreases as $z$ increases, which seems to 
be in contradiction with the increase of shear strength. The point is that the shear strength should not be evaluated only from the actual state of the microstructure but from the potential hardening of the material, which is clearly controlled by the evolution of the anisotropy. A material with high value of $z$ may loose more contacts along the direction of extension without being destabilized, hence developing higher degree of anisotropy. This leads to a high peak strength. At still larger shear deformation, contact gain in the direction of contraction increases and the anisotropy declines to its critical-state value. In this way, the critical state is characterized by a detailed balance between the gain and loss processes of contacts along and perpendicular to the direction of shear strain rate.

\section{RECENT DEVELOPMENTS IN MACROSCOPIC MODELING}

Alongside micromechanical approach, recent progress in macroscopic modeling has mainly focussed on the foundations of classical concepts and models of granular behavior. Among various developments in this context, the authors will present below the thermo-mechanical consistency of models, anisotropy as an independent parameter of the critical state, material instability, statistical physics approach and inertial granular flows.

\section{Thermo-mechanical framework}

Most macroscopic models of granular behavior address correctly the material objectivity and fit a group of experimental data. The objectivity, however, does not ensure the consistency of such models with the framework of thermodynamics along all deformation paths. Indeed, while the thermomechanics of materials has undergone significant developments (Ziegler and Wehrli 1987; Maugin 1992), its application to granular materials is rather recent (Collins 1997; Collins and Hilder 2002; Collins 2005). In this framework, the yield function, flow rule and hardening rules of an elasto-plastic or a hypoplastic theory should be determined from the knowledge of the free energy function $A$ and a dissipation (or dissipation date) function $\Phi$. The work increment is given by $\delta W=\sigma_{i j} d \varepsilon_{i j}=d A+\delta \Phi$ with $\delta \Phi \geq 0$ in general, and $\delta \Phi>0$ when plastic deformations occur. Only $A$ is a state function of elastic and plastic strains. The partial derivatives of $A$ and $\delta \Phi$ with respect to plastic deformations give rise to a back stress and dissipative stress, respectively.

The plastic dissipation is the product of the dissipative stress with the plastic strain increment whereas shift stress dissipates no energy. The back stress is equivalent to kinematic hardening with the displacement of the yield locus. Its physical origin as recoverable plastic work can be attributed 
to "locked-in" elastic strains at the grains scale. This is also consistent with the observation that in granular materials only a weak fraction of frictional contacts slip and dissipate energy (Radjai et al. 1998). More generally, it is suggested that the bimodal nature of stress transmission in granular materials is consistent with this partition and can be used to construct general elasto-plastic models with physical internal variables (Collins and Hilder 2002).

Collins and Hilder showed that most models, including the modified Cam Clay, do involve back stresses and recoverable plastic work. They outlined a general method for the construction of the yield function and flow rule. These laws are first formulated in terms of dissipative stresses with associated flow rule. Then, they are transformed to the true stress space by adding the back stress. This procedure is applied to introduce families of isotropic and anisotropic models which include Mohr-Coulomb and Nor-sand models as special cases (Collins and Hilder 2002). The strengthdilatancy relation is shown to be a consequence of the intimate relation between dilatancy and anisotropy. One interesting feature of this energetic approach is that the flow rule arises from the transformation between dissipative and true stress spaces without requiring a plastic potential.

\section{Accounting for anisotropy in constitutive modeling}

The generic anisotropy of granular texture has been a permanent source of inspiration for improved modeling of granular behavior (Hoque and Tatsuoka 1998; Kruyt and Rothenburg 2004; Dafalias and Manzari 2004; Wan and Guo 2004; Radjai and Roux 2004; Alonso-Marroquin et al. 2005; Li et al. 2012; Zhao and Guo 2013; Gao et al. 2014; Dafalias 2016). In particular, equation (15) clearly shows that the stress ratio is nearly proportional to the contact anisotropy and a phase factor that accounts for the difference between the privileged contact direction and major principal stress direction. If the phase effect were absent, then contact anisotropy would simply be a redundant parameter and could therefore be ignored in macroscopic modeling (the stress ratio playing the same role as contact anisotropy). However, this not the case since for a granular texture obtained along a strain path or through an arbitrary dynamic preparation method such as pouring into a container, the stress state (magnitudes and directions) can change almost instantaneously, and the transient deformations are then controlled by the evolution of the phase difference through the fabric change. Furthermore, not only the stress ratio but also the dilatancy depend on loading direction, a property that is ignored in the conventional CST (M et al. 1998). 
In this way, the directional dependence of mechanical response raises a fundamental issue regarding the definition of the CS, which is classically characterized by a critical value of the stress ratio $\eta_{c}$ and a critical void ratio $e_{c}$ (depending on the ratio of the average stress to a characteristic stress of the material) with no reference to loading direction compared to the fabric state. The issue is the 'dimensionality' of the critical domain in the state parameter space and its uniqueness (Radjai and Roux 2004; Radjai 2009; Li et al. 2012; Zhao and Guo 2013; Dafalias 2016). This point was recently addressed by a subtle Anisotropic Critical State Theory (ACST) (Li et al. 2012; Dafalias 2016). In this theory, the CS is characterized not only by the critical values of stress ratio and void ratio but also by a critical value of fabric anisotropy. Since dilatancy is the most significant parameter of the CST, the combined effect of loading direction and fabric state is formulated in terms of a Dilatancy State Line (DSL), parallel to the Critical State Line (CSL) on the $(e, p)$ plane, which evolves towards the CSL as a function of a scalar anisotropy parameter reflecting the distance of the current fabric state to its critical state. On the other hand, the DSL replaces the CSL in its role of specifying the dilative or contractive state, depending on whether the current void ratio $e$ is smaller or larger than the corresponding void ratio $e_{d}$ at the same $p$. The new state parameter is defined by $\zeta=e-e_{d}=\left(e-e_{c}\right)-\left(e_{d}-e_{c}\right)=\psi-\psi_{A}$, where $\psi$ is the usual state parameter and $\psi_{A}$ represents the anisotropy of the model. The uniqueness and attainability of the CS is proved on the basis of the Gibbs condition of stability. The model is completed by a simple evolution equation for $\psi_{A}$, incorporating the requirement that the latter vanishes in the CS.

The ACST is a general framework within which various constitutive models may be formulated (Gao et al. 2014). It can be considered as a significant step beyond the CST with the advantage of shedding new light on the foundations of the CST and thus paving the way for further developments. The critical anisotropy may be evaluated from a grain-scale statistical approach. In particular, it can be shown that with an appropriate representation of local grain environments, the critical contact anisotropy $a_{c}^{*}$ is related to the values of the coordination number $z^{\max }$ and $z^{\min }$ in the densest and loosest isotropic states, respectively, through a simple relation $a_{c}^{*}=2\left(z^{\max }-z^{\min }\right) /\left(z^{\max }+z^{\min }\right)$ (Radjai 2009; Radjai et al. 2012). 


\section{Higher-order models}

Most elastic-plastic models of granular behavior are local in the sense that the stress at each material point depends only on the strain or strain rate at the same point. Higher-order continuum models have been motivated by the finite size of shear bands, scale effects and particle rotations (Mulhaus and Vardoulakis 1987; Vardoulakis and Aifantis 1991; Vardoulakis and Sulem 1995). By introducing an internal length into the problem, such models remove the ill-posedness of the boundary-value problem arising in softening and shear localization. The internal length scale, which can be attributed either to grain size or the size of mesostructures, accounts for shear band thickness and allows for scale effects to be captured by the theory.

The internal length scales are definitely larger than single grain size $(\sim 10$ grain diameters $)$ as inferred from force-force correlations (see section 3). This force-related length reflects the correlations of the underlying texture and may thus partially depend on boundary conditions and fabric anisotropy, but it remains of the order of several grain diameters (Majmudar and Behringer 2005). However, non-affine velocities reveal much larger lengths involving correlated motions of particles (see section 3) (Radjai and Roux 2002). One highly nontrivial observation is the creeping of grains far away from a moving boundary (the so-called split-bottom geometry) (Nichol et al. 2010). Another well-documented observation is the decrease of the maximum angle of stability of a granular layer as a function of the thickness of the latter (Pouliquen 1999). Recently, S-shaped strain profiles were evidenced in experiments of plane shear flows indicating that the rheology is different close to the walls and its effects extends deeply into the medium (Miller et al. 2013). A local rheology predicts a linear profile when the stress state and all other fabric variables are constant across the flow. A more direct evidence for nonlocal behavior was provided by extensive simulations of Kuhn (Kuhn 2005). By imposing coerced nonuniform shearing, it was shown that the shear stress is strongly affected by both the first and second gradients of shear strain. The same simulations indicate that the dilatancy and particle rotations are not affected by gradients.

The gradient elastic-plastic models have been extensively worked out in the past (Vardoulakis and Aifantis 1991; Goddard 2014). They all involve only the second gradient on the basis that the first gradient is not compatible with the symmetry of the flow. A plastic material length $\ell_{p}$ is introduced as well as a reduced stress rate depending on the Laplacian of the plastic strain rate. The Laplacian accounts for the assumption that the behavior at a given point in the flow depends 
on the nature of the flow in the surrounding region. Recently, Miller et al. used the concept of granulence to introduce an eddy viscosity proportional to the imposed shear rate and squared distance to the walls in order to estimate S-shaped velocity profiles (Miller et al. 2013). Another successful nonlocal model is based on a state parameter field $g$ called granular fluidity (Kamrin and Koval 2012). It enters the rheology through its definition and $\dot{\gamma}=g \mu$ and its value at each material point as a perturbation of its local value $g_{l o c}=\dot{\gamma}_{l o c} / \mu$ by its Laplacian $\xi^{2} \Delta g$ where $\xi$ is a cooperativity length proportional to grain size. This model has shown its ability to quantitatively predict all the above-mentioned nonlocal effects (Kamrin and Henann 2015).

\section{Failure and instabilities}

Instability refers to a state of impossibility of quasi-static monotonic loading so that any infinitesimal load increment leads to dynamic evolution with a burst of kinetic energy or large deformations (Lade 2002; Nicot and Darve 2007). Experimental observations show that the failure is either of geometrical nature as in column buckling, or of material origin as in constitutive behavior. The latter has generally been associated either with vanishing of the determinant of the constitutive tensor as precursor of homogeneous failure at the plastic limit or vanishing of the determinant of the acoustic tensor with the emergence of plastic strain localization. These two criteria coincide for an associative flow rule in which the elasto-plastic tensor is symmetric. But the non-associated behavior of granular materials implies the loss of symmetry so that shear localization may occur before the plastic limit.

Undrained triaxial tests on loose sand show a different type of failure at peak deviatoric stress without shear localization (Nicot et al. 2007). The concepts of controllability and sustainability of equilibrium states were introduced to account for this diffuse failure. Recently, from experiments and DEM simulations it was shown that a whole bifurcation domain in the stress space exists with various possible failure modes (Daouadji et al. 2011). The diffuse failure mode has a directional character with respect to loading. The second-order work $d^{2} W=d \sigma_{i j} d \varepsilon_{i j}$ has been used to analyze failure modes inside the plastic domain. This criterion includes both the plastic limit condition and strain localization, which are special cases of the vanishing of second-order work. The microinstabilities are always present in sheared granular materials in the form of local bursts of individual or collective inertial grain motions but they generally do not propagate to the macro-scale as a result 
of disorder and energy dissipation. They propagate only when they are numerous and organized in such a way that single grain motions are amplified by an avalanche-like process (Staron et al. 2002).

This means that a crucial step for a better understanding of failure is a detailed investigation of the micro-transformations from the contact scale to the system scale, with due considerations of sample size effects. Such processes can be studied, e.g., by deviator stress increments under triaxial conditions. The general picture of the stress-strain behavior is a smooth process when shear deformation is controlled. But when stress increments are applied, a granular material can support finite stress increments without changes in the contact network (and without deformation if grains are rigid) followed by fast rearrangements corresponding to a finite strain step (Combe and Roux 2000; Roux and Combe 2002). With frictionless rigid grains, the finite strain jumps have a Levy statistics, i.e. a power-law distribution of infinite mean - and no deterministic strain can be associated to given stress increments, even in the large system limit (Combe and Roux 2000). Dilatancy vanishes in such systems but a finite shear resistance is observed. Friction modifies this distribution (Roux and Combe 2002), and smooth stress-strain curves are obtained for large samples. The micro-instabilities by which contact networks rearrange and the fabric changes in response to stress variations do not necessarily imply the existence of macro-instabilities (leading, e.g., to strain localization). No specific study has been carried out yet, though, of the dependence of the length scale associated to rearrangements on the proximity to a macroscopic instability criterion except on sandpiles tilted from horizontal towards their angle of repose, indicating that a length scale defined from friction mobilization increases and diverges as the maximum angle of stability is approached (Staron et al. 2002; Staron and Radjai 2005).

\section{Statistical mechanics approach}

Besides kinetic theory of granular gases, an increasing number of researchers have dealt with granular materials by means of concepts and theoretical tools of statistical mechanics. In such attempts, granular media are very often used as a metaphor of amorphous materials such as colloids, foams and glasses. For example, the SOC is a perfect model of out-of-equilibrium systems in which sandpiles are used for illustration (Bak et al. 1988).

One of the earliest statistical models of granular matter is Edwards' volume ensemble (Edwards 
and Oakeshott 1989). Since the volume (or packing fraction) of a granular material can change, Edwards suggested that a microcanonical granular ensemble may be defined by all jammed mechanically stable states of equal volume (replacing energy). Hence, a statistical mechanical model can be built by assuming equiprobability of all such states. In particular, a "compactivity" variable can be introduced as the derivative of the volume with respect to entropy (analogous to thermodynamical temperature). There have been attempts to measure the volumetric entropy and compactivity of simple granular systems from the distribution of volumes of tessellation cells (Aste and DiMatteo 2008). This has been further investigated by simulations and implies that the compactivity is a measure of void ratio (Oquendo et al. 2016). A dual approach consists in considering the force ensemble as the set of all force configurations with a given confining pressure (Snoeijer et al. 2004).

The statistical entropy has more generally been employed to rationalize force distributions and local structures such as the distributions of coordination numbers, void valences and porosities (Bagi 1997; Kruyt and Rothenburg 2002b; Goddard 2004; Troadec et al. 2002; Aste et al. 2005; Kuhn 2014). Given a distribution $P_{i}$, maximizing the entropy $S=-\sum_{i} P_{i} \log P_{i}$ for a set of constraints is equivalent to searching the most unbiased solution with respect to the missing information. The results obtained by this method with a proper account of constraints are often in excellent agreement with numerically simulated measures in the critical state for which meaningful statistics can be extracted. But whether and why the critical state should be a maximally disordered state (in the sense of the amount of missing information) is still a matter of discussion. One still needs to show that the entropy is lower below and above the critical state.

Jamming transition, i.e. the arrest of particles from a dynamic state, has attracted considerable work (Liu and Nagel 1998). A supercooled liquid turns into a glass with a finite yield stress as the temperature is lowered. This process is very similar to the arrest of shaken grains in a packing of finite yield stress when the shaking intensity is decreased or the packing fraction is lowered (Jaeger 2015). Such transitions may be described by the same jamming phase diagram with temperature, packing fraction and pressure as control variables. Granular materials have been extensively used as model system for the investigation of this jamming diagram.

Static disordered packings of spheres have been a subject of great interest for their geometric properties, which are relevant for all kinds of amorphous materials, made of atoms, colloidal particles or macroscopic grains (Aste and Weaire. 2000; Aste et al. 2005; Torquato 2010). In 
the absence of friction, rigid grains generically assemble under confining forces into configurations devoid of force indeterminacy (i.e., given the geometry of the contact network, contact forces are entirely determined by the external load and the equilibrium requirement). This sets upper bounds on coordination numbers, which still apply to frictional grains. With spherical grains, isostaticity is achieved (no displacement indeterminacy or "floppy mode" (Roux 2000; Torquato 2010) exists on the force-contact network), which results in a coordination number equal to 6 if the "rattler" grains that carry no force are excluded. Without friction, those configurations - often termed jammed in the recent literature - are local minima of the potential energy of external forces (Roux 2000). If subjected to an isotropic state of stress, they realize a local maximum of solid fraction in configuration space - whence the identification (O'Hern et al. 2003; Agnolin and Roux 2007a) of the random close packing (RCP) state with such isotropically confined assemblies of rigid frictionless objects, provided it can be regarded as uniquely defined - all volume-minimizing configurations, if quickly assembled, sharing the same solid fraction in the limit of large systems. This latter condition appears to be satisfied for rapidly assembled packings (Agnolin and Roux 2007a), in which slow evolutions (toward crystallization for identical beads) or, possibly, towards separation for mixtures, do not take place (lack of uniqueness for slower protocols is shown in (Chaudhuri et al. 2010)).

The absence of dilatancy reported for frictionless objects (Peyneau and Roux 2008a; Azéma et al. 2015) could partly explain that the same maximum density is recorded in various situations of packings assembled quickly, but in such a way that the mobilization of friction is circumvented: the stress state needs not be isotropic. The concept of the RCP, thus related to a mechanical definition, was however criticized as somewhat ill-defined by some authors (Torquato 2010), who adopted instead a notion of a Maximally Random Jammed (MRJ) packing of hard particles. The MRJ state minimizes some order metric under a condition of jamming. A discussion of jamming (stability of equilibrium state under the given external load) for arbitrary-shaped, frictional grains is provided in (Bagi 2007).

\section{Inertial granular flows}

Most modeling attempts mentioned above concern "quasi-static" deformations of granular materials, which are assumed be rate-independent. However, the flow of granular matter is common in the transport of granular materials such as minerals and cereals, as well as at larger scales of 
rock avalanches, debris flows and other geological surface processes. Bagnold was the first to model granular flows by considering grains of average size $d$ and density $\rho_{s}$ subjected to a controlled shear flow of rate $\dot{\gamma}$ at constant volume. With these parameters, a dimensional analysis implies that the shear stress $\tau$ is propotional to $\rho_{s} d^{2} \dot{\gamma}^{2}$ with a prefactor depending on the packing fraction $\phi$ (Bagnold 1954).

For a long time, the scaling of shear stress as $\dot{\gamma}^{2}$ was considered to be a consequence of momentum exchange whose average value and frequency are proportional to the shear rate. This scaling was incorporated in a general theory of "rapid granular flows" but its applicability to dense granular flows remained questionable as most flows encountered in practice are both frictional and collisional (Hutter and Scheiwiller 1983; Campbell 1990). The general theory of collisional granular materials was developed using the classical formalism of kinetic theory and by introducing a granular temperature $T=\left\langle\delta v^{2}\right\rangle$, the mean square of nonaffine (or fluctuating) particle velocities, and by introducing a dissipation term through restitution coefficients for mass conservation (Jenkins and Savage 1983). In this theory, all stresses are of kinetic origin. It should also be recalled that reference physical system in both rapid flows and granular gases has been constant-volume shear and the rheology is described by normal and shear viscosities $\eta_{n}$ and $\eta_{t}$, respectively, defined by $p=\eta_{n} \dot{\varepsilon}_{t}$ and $\tau=\eta_{t} \dot{\varepsilon}_{t}$, where $\dot{\varepsilon}_{t}$ is the shear rate.

It was only in 2004 that it became clear that frictional and inertial flows can be described in the same framework if the volume as control parameter is replaced by the confining pressure $p$ (GDR-MiDi 2004). The relevant dimensionless number is then the inertial number $I$ :

$$
I=\dot{\gamma} d\left(\frac{\rho_{s}}{p}\right)
$$

This number is the ratio of the internal relaxation time of the grains $d\left(\rho_{s} / p\right)$ to the shear time $1 / \dot{\gamma}$. At low values of $I$, typically below $10^{-3}$, the flow is rate-independent. At higher values of $I$, the bulk friction coefficient $\mu$ increases with $I$ whereas the packing fraction decreases. Phenomenological laws $\mu(I)$ and $\phi(I)$ for steady granular flows in combination with continuum conservation equations correctly predict the velocity and stress fields in various flow geometries (da Cruz et al. 2005; Jop et al. 2006). The increase of $\mu$ with $I$ despite an increasingly lower packing fraction reveals a genuine microstructure. As $I$ increases, the force chains become more sparse, the correlation length 
of connected particles decrease, the contact lifetimes decline, and an increasing number of impulsive forces and frictionally mobilized contacts come into play. Azema and Radjai applied stress partition, as in quasi-static deformations (see section 4), to show that the main mechanism for the increase of $\mu$ is contact anisotropy (Azéma and Radjai 2014).

In the $I$-based rheology, $\mu(I)$ and $\phi(I)$ are the main variables. However, using the definition of $I$, it is straightforward to obtain the $\phi$-based rheology (constant volume) with $\eta_{n}=I^{-2}$ and $\eta_{t}=\mu I^{-2}$. Using the data, it is shown that both viscosities diverge as $\left(\phi_{c}-\phi\right)^{-\alpha}$, with $\alpha \simeq 2$, when $I \rightarrow 0$, corresponding to $\phi \rightarrow \phi_{c}$ (Boyer et al. 2011). The value of $\phi_{c}$ is $\simeq 0.58$ for spherical particles of the same size and coincides with the CS packing fraction at low confining pressures. A similar divergence is observed in dense suspensions. This indicates that the same framework may be used to unify dense granular flows and dense viscous suspensions by accounting for viscous forces as well as inertial and frictional forces.

\section{CONCLUSIONS: EXPANDING FRONTIERS}

The fast expansion of granular science does not yet seem to converge to a common denominator. While new frontiers are constantly explored, there remain gaps and barriers that have been dodged or left for future. The developments have been driven by so many problems of practical interest, increasingly resolved measurements and imaging techniques, DEM simulations of increasing computational efficiency and fruitful analogy with glassy materials. The simple packing of equal spheres has grown into a complex conceptual object in which the frontiers between trivial and fundamental are sometimes confused. But these are only signs of an explosive expansion of the field.

Relating the macroscopic behavior of granular materials to their microstructure is a longstanding dream, which has not yet been realized. A long path remains towards a multiscale approach based on the details of granular microstructure. For example, there is still only a partial understanding of relevant fabric variables and no general model for their evolution. Many models consider only simple loading paths in which a single scalar state parameter may just be enough. The role of mesoscale structures and their correlations also remain to be clarified. Advanced constitutive models can account for complex loading paths but their state variables are not explicitly connected with variables pertaining to the microstructure. On the other hand, the particle-scale variables such as forces and mobilized contacts have broad and scale-dependent distributions which 
are often well characterized from simulations and experiments but they elude statistical modeling.

In the same way, despite decisive progress made on inertial granular flows, the empirical expressions of shear stress and packing fraction as a function of the inertial number have no theoretical basis. Although nonlocal effects have been evidenced by experiments and simulations and captured by models based on higher gradients of strain or a state parameter (as in the fluidity model), a pending question remains as to the nature of fluidity and some observations such as the dependence of the stress on the first strain gradient. It is important to note that the continuum equations assume a representative volume element. The nonlocal effects refer mostly to the grain size, which is clearly not a representative size of the material behavior. Moreover, the perturbations introduced by the boundary walls in the microstructure and their long-range correlations together with a local model thoroughly based on the microstructure may allow replacing nonlocal models to explain such "nonlocal" effects. These aspects clearly need further investigation.

An important aspect of granular materials concerns particle geometrical properties such as their shape and size distributions. Such geometrical effects are crucial in many applications and they raise fundamental questions with respect to shear strength, dilatancy, stability and flow of granular materials. For example, the packing fraction is not a monotonous function of the particle shape as it deviates from a sphere; see Fig. 6. A generic shape parameter may be introduced to capture this effect of asphericity, but second-order shape parameters are also important for shear strength and space-filling properties of particles. This is a vast field that only begins to be investigated in a systematic fashion. The shape and size effects need enhanced experimental and numerical skills. The reason is that particle shapes cannot easily be controlled and numerical simulations require more advanced techniques of contact detection and much larger numbers of particles as size polydispersity and shape variability in samples increases.

3D printing of particles opens the way for systematic experimental research along these lines. Particles of nonconvex and other exotic shapes can thus be produced. A packing of such particles is governed by real interlocking of the particles leading to a "geometric cohesion" (Franklin 2012). Star-shaped, Z-shaped and similar particles spontaneously jam in stable structures of high shear strength when poured into a container. Hence, optimizing particle shape may allow one to design structures that can emerge from random jamming of particles. This is what Jaeger calls "jamming by design" as a "process that gets us from desired properties to requirements for the constituent 
components" (Jaeger 2015; Reis et al. 2015). Packings of nonconvex aggregates of overlapping spheres can be optimized to create the densest packing (Roth and Jaeger 2016). This is not only of immense interest to the design of special microstructures in sintered powders combining high permeability, strength and manageability, for example, but also to the architecture and industrial design communities to create innovative structures.

Besides particle geometry, there is presently a vast scope for research on several other aspects that were omitted from this review. The topic of cohesive granular materials and their flow behavior has been much less at the focus of recent research. Cohesive interactions may be due to solid surface forces as in fine powders or a consequence of capillary forces as in unsaturated soils. The mixing process of a powder with a liquid, distribution of liquid clusters, packing states and their rheology have only recently been investigated at the particle scale. The agglomeration process in applications to powders in food science and iron ores in steel-making industry is complex as it involves both cohesive interactions and inertial flows.

Granular materials composed of crushable particles and their behavior while the particles can break under evolving load and/or deformation have been mostly investigated in connection with soils and rockfills. Particle breakage affects the stress-strain behavior as a result of the evolution of particle size distribution, which affects in turn the dilatancy and hence shear strength (Daouadji et al. 2001; Einav 2007; Russell et al. 2009; Daouadji and Hicher 2010). Other effects concern the evolution of particle shapes and their frictional contacts that induce nontrivial effects. Such evolving granular materials have often been modeled by assuming that each particle is an aggregate of smaller particles. However, to avoid finite size effects, it is necessary to consider larger aggregates and large packings of aggregates in order to be able to extract useful information from such discrete models.

Another area of research, remaining almost fully unexplored, is that of ultra-soft particles. In hard granular materials, the elastic deformations are assumed to be concentrated at the contact points, and thus described as a function of the rigid-body degrees of freedom of the particles. This approximation is too crude in many applications, and the particles undergo large deformations (Nezamabadi et al. 2015). Metallic powders, for example, deform plastically without rupture. Likewise, many pharmaceutical and food products are soft-particle materials. The particle shape change occurs also in clays, which are composed of nano-scale aggregates. Such materials can 
undergo volume change by particle shape and size change under moderate external load with enhanced space filling properties. The compaction and shear behavior beyond this "jamming" limit require fundamental research effort in the future. Proper simulation methods, by coupling a continuum description of the particles, with the Material Point Method that can allow for large deformations, for example, coupled with contact dynamics method for the treatment of contacts between deformable particles, need to be developed.

Although only the modeling aspects were at the focus of this paper, let us recall here that the research on granular materials has been developed from increasingly precise experimental techniques to measure particle displacement fields and forces (Lesniewska and Wood 2009). The development of new measurement techniques and their applications to various granular experiments can be a subject of a stand-alone review paper. Automated simultaneous measurements of applied stresses and strains in arbitrary directions are already in use in soil mechanics. The experimental challenge has been so far the perfect control of boundary conditions and homogeneity of samples. However, the challenge has now shifted to the measurement of fabric variables in $3 \mathrm{D}$ packings of particles by resolving contacts between particles. Today, high-resolution X-ray computed tomography can provide access to this information (Andò et al. 2012). 3D photoelastic imaging of forces is currently being developed (Brodu et al. 2015), and it will be a valuable tool for accessing normal and tangential forces and their evolution. The ultimate experiment for future will be simultaneous measurements of particle displacements and contact forces in 3D with variable boundary conditions.

The authors thank Mahdi Taeibat for fruitful discussions. Farhang Radjai would like to acknowledge the support of the ICoME2 Labex (ANR-11-LABX-0053) and the A*MIDEX projects (ANR-11-IDEX-0001-02) cofunded by the French program Investissements d'Avenir, managed by the French National Reseach Agency (ANR). 


\section{APPENDIX I. REFERENCES}

Agnolin, I. and Roux, J.-N. (2007a). "Internal states of model isotropic granular packings. I. Assembling process, geometry, and contact networks.." Phys Rev E, 76(6-1), 061302.

Agnolin, I. and Roux, J.-N. (2007b). "Internal states of model isotropic granular packings. II. Compression and pressure cycles.." Phys Rev E, 76(6-1), 061303.

Agnolin, I. and Roux, J.-N. (2007c). "Internal states of model isotropic granular packings. III. Elastic properties.." Phys Rev E, 76(6-1), 061304.

Alonso-Marroquin, F. and Herrmann, H. J. (2002). "Calculation of the incremental stress-strain relation of a polygonal packing." Phys. Rev. E, 66(2), 021301-.

Alonso-Marroquin, F., Luding, S., Herrmann, H. J., and Vardoulakis, I. (2005). "Role of anisotropy in the elastoplastic response of a polygonal packing." Phys. Rev. E, 71(5), 051304.

Andò, E., Hall, S. A., Viggiani, G., Desrues, J., and Bésuelle, P. (2012). "Experimental micromechanics: grain-scale observation of sand deformation." Géotechnique Letters, 2, 107-112.

Andrade, J., Avila, C., Hall, S., Lenoir, N., and Viggiani, G. (2011). "Multiscale modeling and characterization of granular matter: From grain kinematics to continuum mechanics." Journal of the Mechanics and Physics of Solids, 59, 237-250.

Andrade, J. E. and Tu, X. (2009). "Multiscale framework for behavior prediction in granular media." Mechanics of Materials, 41, 652-669.

Antony, S. J. (2001). "Evolution of force distribution in three-dimensional granular media.." Phys. Rev. E, 63(1 Pt 1), 011302.

Aste, T. and DiMatteo, T. (2008). "Emergence of gamma distributions in granular materials and packing models." Phys. Rev. E, 77, 021309.

Aste, T., Saadatfar, M., and Senden, T. J. (2005). "The geometrical structure of disordered sphere packings." Phys. Rev. E, 71, 061302.

Aste, T. and Weaire., D. (2000). The Pursuit of Perfect Packing. Institute of Physics Publishing.

Azéma, E. and Radjai, F. (2014). "Internal structure of inertial granular flows." Phys. Rev. Lett., 112(7), 078001-.

Azéma, E., Radjai, F., Peyroux, R., and Saussine, G. (2007). "Force transmission in a packing of pentagonal particles.." Phys. Rev. E, 76(1 Pt 1), 011301. 
Azéma, E., Radjaï, F., and Roux, J.-N. (2015). "Internal friction and absence of dilatancy of packings of frictionless polygons." Phys. Rev. E, 91, 010202(R).

Azema, E., Radjai, F., Saint-Cyr, B., Delenne, J.-Y., and Sornay, P. (2013). "Rheology of 3d packings of aggregates: microstructure and effects of nonconvexity." Phys. Rev. E, 87, 052205.

Azéma, E., Saussine, G., and Radjai, F. (2009). "Quasistatic rheology, force transmission and fabric properties of a packing of irregular polyhedral particles." Mechanics of Materials, 41, 729-741.

Bagi, K. (1997). "Analysis of micro-variables through entropy principle." Powders and Grains 1997, R. Behringer and J. T. Jenkins, eds., Balkema, Rotter-dam, 251-254.

Bagi, K. (2007). "On the Concept of Jammed Configurations from a Structural Mechanics Perspective." Granular Matter, 9, 109-134.

Bagnold, R. A. (1954). "Experiments on a gravity-free dispersion of large solid spheres in a newtonian fluid under shear." Proc. Royal Soc. London, 225, 49-63.

Bak, P., Tang, C., and Wiesenfeld, K. (1987). "Self-organized criticality: An explanation of $1 / f$ noise." Phys. Rev. Lett., 59(4), 381-384.

Bak, P., Tang, C., and Wiesenfeld, K. (1988). "Self-organized criticality." Phys. Rev. A, 38(1), $364-375$.

Bardet, J. P. (1994). "Observations on the effects of particle rotations on the failure of idealized granular materials." Mechanics of Materials, 18, 159-182.

Bathurst, R. J. and Rothenburg, L. (1988). "Micromechanical aspects of isotropic granular assemblies with linear contact interactions." J. Appl. Mech., 55, 17.

Been, K. and Jefferies, M. (1985). "A state parameter for sands." Géotechnique, 35, 99-112.

Bernal, J. D. (1960). "Geometry and the structure of monatomic liquids." Nature, 185, 68-70.

Berryman, J. G. (1986). "Random close packing of hard spheres and disks." Phys. Rev. A, 27, 1053.

Biarez, J. (1962). "Contribution à l'étude des propriétés mécaniques des sols et des matériaux pulvérulents." Ph.D. thesis, Grenoble, France, Grenoble, France.

Bolton, M. D. (1986). "The strength and dilatancy of sands." Géotechnique, 36, 65-78.

Boyer, F., Guazzelli, E., and Pouliquen, O. (2011). "Unifying suspension and granular rheology." Phys. Rev. Lett., 107, 18.

Brodu, N., Dijksman, J. A., and Behringer, R. P. (2015). "Spanning the scales of granular materials through microscopic force imaging." Nature Communications, 6:6361. 
Cambou, B. (1993). "From global to local variables in granular materials." Powders and Grains 93, C. Thornton, ed., Amsterdam, A. A. Balkema, 73-86.

Campbell, C. S. (1990). "Rapid granular flows." Annu. Rev. Fluid Mech., 22, 57.

Casagrande, A. (1936). "Characteristics of cohesionless soils affecting the stability of slopes and earth fills." J. Boston Soc. Civil Eng., 23, 257-276.

Cates, M. E., Wittmer, J. P., Bouchaud, J.-P., and Claudin, P. (1998). "Jamming, force chains, and fragile matter." Phys. Rev. Lett., 81(9), 1841-1844.

Chang, C. S. and Bennett, K. (2015). "Micromechanical modeling for the deformation of sand with noncoaxiality between the stress and material axes." Journal of Engineering Mechanics, C4015001.

Chang, C. S. and Hicher, P.-Y. (2005). "An elasto-plastic model for granular materials with microstructural consideration." International Journal of Solids and Structures, 42, 4258-4277.

Chang, C. S., Hicher, P. Y., and Daouadji, A. (2009). "Investigating instability in granular materials by means of a micro-structural model." European Journal of Environmental and Civil Engineering, 13, 167-186.

Chang, C. S. and Misra, A. (1990). "Application of uniform strain theory to heterogeneous granular solids." Journal of Engineering Mechanics, 116, 2310-2328.

Chang, C. S., Yin, Z.-Y., and Hicher, P.-Y. (2011). "Micromechanical analysis for interparticle and assembly instability of sand." Journal of Engineering Mechanics, 137, 155-168.

Chaudhuri, P., Berthier, L., and Sastry, S. (2010). "Jamming Transitions in Amorphous Packings of Frictionless Spheres Occur over a Continuous Range of Volume Fractions." Phys. Rev. Lett., 104(16).

Christoffersen, J., Mehrabadi, M. M., and Nemat-Nasser, S. (1981). "A micromechanical description of granular material behavior." J. Appl. Mech., 48, 339-344.

Collins, I. F. (1997). "The use of legendre transformations in developing the constitutive laws of geomechanics from thermodynamic principles." IUTAM Symposium on Mechanics of Granular and Porous Materials, N. A. Fleck and A. C. E. Cocks, eds., Kluwer Academic Publishers, 151159.

Collins, I. F. (2005). "Elastic/plastic models for soils and sands." International Journal of Mechanical Sciences, 47(4-5), 493-508. 
Collins, I. F. and Hilder, T. (2002). "A theoretical framework for constructing elastic/plastic constitutive models of triaxial tests." Int. J. Numer. Anal. Meth. Geomech., 26, 1313-1347.

Combe, G., Richefeu, V., and Stasiak, M. (2015). "Experimental validation of a nonextensive scaling law in confined granular media." Phys. Rev. Lett., 115, 238301.

Combe, G. and Roux, J.-N. (2000). "Strain versus stress in a model granular material: A devil's staircase." Phys. Rev. Lett., 85(17), 3628-3631.

Coppersmith, S. N., Liu, C., Majumdar, S., Narayan, O., and Witten, T. A. (1996). "Model for force fluctuations in bead packs." Phys. Rev. E, 53(5), 4673-4685.

Coulomb, C. A. (1773). "Essai sur un application de règles de maximis et minimis à quelques poblèmes de staique relatifs à l'architcture." Acad. R. Sci. Mem. Math. Phys. Acad. R. Sci., Paris, 7, 343-382.

Coulomb, C. A. (1781). "Théorie des Machines Simples." Academie des Sciences, 10, 166.

Coumoulos, D. G. (1967). A radiographic study of soils. University of Cambridge.

Cundall, P. A. and Strack, O. D. L. (1979). "A discrete numerical model for granular assemblies." Géotechnique, 29(1), 47-65.

Cundall, P. A. and Strack, O. D. L. (1983). "Modeling of microscopic mechanisms in granular materials." Mechanics of Granular Materials: New Models and Constitutive Relations, J. T. Jenkins and M. Satake, eds., Amsterdam, Elsevier, 137-149.

da Cruz, F., Emam, S., Prochnow, M., Roux, J.-N., and cois Chevoir, F. (2005). "Rheophysics of dense granular materials: discrete simulation of plane shear flows.." Phys. Rev. E, 72(2 Pt 1), 021309.

Dafalias, Y. (2016). "Must critical state theory be revisited to include fabric effects?." Acta Geotechnica, 11, 479-491.

Dafalias, Y. F. and Manzari, M. T. (2004). "Simple plasticity sand model accounting for fabric change effects." Journal of Engineering Mechanics, 130, 622-634.

Dantu, P. (1957). "Contribution à l'étude mécanique et géométrique des milieux pulvérulents." Proc. Of the 4th International Conf. On Soil Mech. and Foundation Eng., Vol. 1, London, Butterworths Scientific Publications, 144-148.

Daouadji, A., Darve, F., Gali, H. A., Hicher, P. Y., Laouafa, F., Lignon, S., Nicot, F., Nova, R., Pinheiro, M., Prunier, F., Sibille, L., and Wan, R. (2011). "Diffuse failure in geomaterials: Ex- 
periments, theory and modelling." International Journal for Numerical and Analytical Methods in Geomechanics, 35, 1731-1773.

Daouadji, A. and Hicher, P. Y. (2010). "An enhanced constitutive model for crushable granular materials." Int. J. Num. Anal. Methods in Geomechanics, 34, 555-580.

Daouadji, A., Hicher, P. Y., and Rahma, A. (2001). "Modelling grain breakage influence on mechanical behaviour of granular media." European Journal of Mechanics, A/solids, 20, 113-137.

Darve, F. and Laouafa, F. (1999). "Plane strain instabilities in soil: Application to slopes instability." Numerical Models in Geomaterials, Prande, Pietruszczak, and Schweiger, eds., Rotterdam, Balkema, 85-90.

Darwin, G. H. (1883). "On the horizontal thrust of a mass of sand." Minutes of the Proceedings Instituation of Civil Engineering, 350-378.

Desrues, ., Lanier, J., and Stutz, P. (1983). "Localization of deformation in tests on sand samples." Eng. Fracture Mechanics, 21, p909-921.

Desrues, J., Chambon, R., Mokni, M., and Mazerolles, F. (1996). "Void ratio evolution inside shear band in triaxial sand specimens studied by computed tomography." Géotechnique, 46, 529-546.

Donev, A., Cisse, I., Sachs, D., Variano, E. A., Stillinger, F. H., Connelly, R., Torquato, S., and Chaikin, P. M. (2004). "Improving the density of jammed disordered packings using ellipsoids.." Science, 303(5660), 990-993.

Donev, A., Torquato, S., and Stillinger, F. H. (2005). "Pair correlation function characteristics of nearly jammed disordered and ordered hard-sphere packings.." Phys Rev E Stat Nonlin Soft Matter Phys, 71(1 Pt 1), 011105.

Drescher, A. and de Josselin de Jong, G. (1972). "Photoelastic verification of a mechanical model for the flow of a granular material." J. Mech. Phys. Solids, 20, 337-351.

Duttine, A., Di Benedetto, H., Pham Van Bang, D., and Ezaoui, A. (2007). "Anisotropic small strain elastic properties of sands and mixture of sand-clay measured by dynamic and static methods." Soils and Foundations, 47(3), 457-472.

E., B. (1996). "Calibration of a comprehensive constitutive equation for granular material." Soils Found., 36, 13-26.

Edwards, S. F. and Oakeshott, R. B. S. (1989). "Theory of powders." Physica A, 157, 1080.

Einav, I. (2007). "Breakage mechanics, part i: theory." Journal of the Mechanics and Physics of 
Solids, 55, 1274-1297.

Einav, I. (2012). "The unification of hypo-plastic and elasto-plastic theories." International Journal of Solids and Structures, 49, 1305-1315.

Estrada, N., Taboada, A., and Radjai, F. (2008). "Shear strength and force transmission in granular media with rolling resistance." Phys. Rev. E, 78.

Franklin, S. V. (2012). "Geometric cohesion in granular materials." Physics Today.

Fuentes, W., Triantafyllidis, T., and Lizcano, A. (2012). "Hypoplastic model for sands with loading surface." Acta Geotechnica, 7, 177-192.

Gao, Z., Zhao, J., Li, X.-S., and Dafalias, Y. F. (2014). "A critical state sand plasticity model accounting for fabric evolution." Int. J. Numer. Anal. Meth. Geomech., 38, 370-390.

GDR-MiDi (2004). "On dense granular flows." Eur. Phys. J. E, 14, 341-365.

Geoffroy, H., di Benedetto, H., Duttine, A., and Sauzéat, C. (2003). "Dynamic and cyclic loadings on sands: results and modelling for general stress-strain conditions." Deformation characteristics of geomaterials, H. diBenedetto, T. Doanh, H. Geoffroy, and C. Sauzéat, eds., Lisse, Swets and Zeitlinger, 353-363.

Goddard, J. (2004). "On entropy estimates of contact forces in static granular assemblies." International Journal of Solids and Structures, 41(21), 5851-5861.

Goddard, J. (2014). "Continuum modeling of granular media." Applied Mechanics Reviews, 66, 050801.

Goddard, J. D. (1990a). "Nonlinear elasticity and pressure-dependent wave speeds in granular media." Proc. R. Soc. Lond. A, 430, 105.

Goddard, J. D. (1990b). "Nonlinear elasticity and pressure-dependent wave speeds in granular media." Proc. Roy. Soc. London, 430, 105-131.

Goldhirsch, I. and Zanetti, G. (1993). "Clustering instability in dissipative gases." Phys. Rev. Lett., 70(11), 1619-1622.

Grammenoudis, P. and Tsakmakis, C. (2005). "Finite element implementation of large deformation micropolar plasticity exhibiting isotropic and kinematic hardening effects." Numerical Methods in Engineering, 62, 1691-1720.

Gudehus, G. S. F. (1996). "A comprehensive constitutive equation for granular materials." Soils Found., 36, 1-12. 
Guo, N. and Zhao, J. (2016). "Parallel hierarchical multiscale modelling of hydro-mechanical problems for saturated granular soils." Comput. Methods Appl. Mech. Engrg., 305, 37-61.

Hashiguchi, K. (1979). "A derivation of the associated flow rule." J. Fac Agric. Kyushu Univ., 24, $75-80$.

Hashiguchi, K. and Chen, Z. P. (1998). "Elastoplastic constitutive equation of soils with the subloading surface." Int. J. Numer. Anal. Methods Geomech., 22, 197-227.

He, Q. C. (2014). "On the micromechanical definition of macroscopic strain and strain-rate tensors for granular materials." Computational Materials Science, 94, 51-57.

Hicher, P.-Y. (1996). "Elastic properties of soils." ASCE Journal of Geotechnical Engineering, 122, 641-648.

Hoque, E. and Tatsuoka, F. (1998). "Anisotropy in elastic deformation of granular materials." Soils and Foundations, 38, 163-179.

Huang, W., Sloan, S., and Fityus, S. (2008). "Incorporating a predefined limit condition in a hypoplastic model by means of stress transformation." Mechanics of Materials, 40, 796-802.

Huang, W.-X., Wu, W., Sun, D.-A., and Sloan, S. (2006). "A simple hypoplastic model for normally consolidated clay." Acta Geotechnica, 1, 15-27.

Hutter, K. and Scheiwiller, T. (1983). "Rapid plane flow of granular materials down a chute." Mechanics of granular media - New models and constitutive relations, Amsterdam, Elsevier.

Jaeger, H. and Nagel, S. (1996). "Granular solids, liquids and gases." Reviews of Modern Physics, $68,1259-1273$.

Jaeger, H. M. (2015). "Celebrating soft matter's 10th anniversary: Toward jamming by design." Soft Matter, 11, 12-27.

Jaeger, H. M., Liu, C., Nagel, S. R., and Witten, T. A. (1990). "Flow in granular materials: Self-organized non-critical behavior." Relaxation and Related Topics in Complex Systems, A. Campbell and C. Giovannella, eds., Plenum Press, London, 235.

Jefferies, M. G. (1993). "Nor-sand : a simple critical state model for sand." Géotechnique, 43, 91-103.

Jefferies, M. G. and Been, K. (2006). Soil Liquefaction: A Critical State Approach. Taylor \& Francis, London.

Jenkins, J. T. and Richman, M. W. (1985). "Kinetic theory for plane flows of a dense gas of 
identical, rough, inelastic, circular disks." Phys. of Fluids, 28, 3485-3494.

Jenkins, J. T. and Savage, S. B. (1983). "A theory for the rapid flow of identical, smooth, nearly elastic, spherical particles." J. Fluid Mech., 130, 187-202.

Jia, X., Caroli, C., and Velický, B. (1999). "Ultrasound propagation in externally stressed granular media." Phys. Rev. Lett., 82, 1863-1866.

Johnson, K. L. (1985). Contact Mechanics. Cambridge University Press.

Jop, P., Forterre, Y., and Pouliquen, O. (2006). "A constitutive law for dense granular flows.." Nature, 441(7094), 727-730.

Jullien, R., Meakin, P., and Pavlovitch, A. (1992). "Random packings of spheres built with sequential models." J. of Phys. A, 25, 4103.

Kamrin, K. and Henann, D. L. (2015). "Nonlocal modeling of granular flows down inclines." Soft Matter, 2015, 11, 179-185.

Kamrin, K. and Koval, G. (2012). "Nonlocal constitutive relation for steady granular flow." Phys. Rev. Lett., 108, 178301.

Kaneko, K., Terada, K., Kyoya, T., and Kishino, Y. (2003). "Global-local analysis of granular media in quasi-static equilibrium." International Journal of Solids and Structures, 40, 4043-4069.

Katagiri, J., Matsushima, T., and Yamada, Y. (2010). "Simple shear simulation of 3d irregularlyschaped particles by image-based dem." Granular Matter, 12, 491-497.

Kolymbas, D. (1991). "An outline of hypoplasticity." Arch. App. Mech., 61, 143-154.

Kolymbas, D., Herle, I., and von Wolffersdorff, P. A. (1995). "Hypoplastic constitutive equation with internal variables." Int. J. for Numerical and Analytical Methods in Geomechanics, 19(XXX), 415-436.

Kruyt, N. P. and Rothenburg, L. (1996). "Micromechanical definition of strain tensor for granular materials." ASME Journal of Applied Mechanics, 118, 706-711.

Kruyt, N. P. and Rothenburg, L. (2002a). "Micromechanical bounds for the effective elastic moduli of granular materials." International Journal of Solids and Structures, 39, 311-324.

Kruyt, N. P. and Rothenburg, L. (2002b). "Probability density functions of contact forces for cohesionless frictional granular materials." International Journal of Solids 8 Structures, 39, 571583.

Kruyt, N. P. and Rothenburg, L. (2004). "Kinematic and static assumptions for homogenization in 
micromechanics of granular materials." Mechanics of Materials, 36(12), 1157-1173.

Kuhn, M. R. (1999). "Structured deformation in granular materials." Mech. Mater., 31, 407-429.

Kuhn, M. R. (2005). "Are granular materials simple? an experimental study of strain gradient effects and localization." Mechanics of Materials, 37, 607-627.

Kuhn, M. R. (2014). "Dense granular flow at the critical state: maximum entropy and topological disorder." Granular Matter, 16, 499-508.

Kuhn, M. R. and Bagi, K. (2004). "Contact rolling and deformation in granular media." International Journal of Solids and Structures, 41, 5793-5820.

Kuwano, R. and Jardine, R. J. (2002). "On the applicability of cross-anisotropic elasticity to granular materials at very small strains." Géotechnique, 52, 727-749.

La Ragione, L. and Jenkins, J. T. (2007). "The initial response of an idealised granular material." Proceedings of the Royal Society A, 463, 735-758.

La Ragione, L. and Magnanimo, V. (2012a). "Contact anisotropy and coordination number for a granular assembly: A comparison of distinct-element-method simulations and theory." Phys. Rev. E, 031304 .

La Ragione, L. and Magnanimo, V. (2012b). "Evolution of the effective moduli of an anisotropic, dense, granular material." Granular Matter, 14, 749-757.

Lade, P. V. (1994). "Instability and liquefaction of granular materials." Computers and Geotechnics, $16,123-151$.

Lade, P. V. (2002). "Instability, shear banding, and failure in granular materials." International Journal of Solids and Structures, 39, 3337-3357.

Lanier, J., Caillerie, D., Chambonn, R., Viggiani, G., B!esuelle, P., and Desrues, J. (2004). "A general formulation of hypoplasticity." Int. J. Numer. Anal. Meth. Geomech., 28, 1461-1478.

Lesniewska, D. and Wood, D. M. (2009). "Observations of stresses and strains in a granular material." Journal of Engineering Mechanics, 135, 1038-1054.

Li, X. and Dafalias, Y. (2000). "Dilatancy for cohesionless soils." Geotechnique, 50, 449-460.

Li, X. and Dafalias, Y. (2015). "Dissipation consistent fabric tensor definition from dem to continuum for granular media." Journal of the Mechanics and Physics of Solids, 78, 141-153.

Li, X. S., , and Dafalias, Y. F. (2012). "Anisotropic critical state theory: Role of fabric." J. Eng. Mech., 138, 263-275. 
Liu, A. J. and Nagel, S. R. (1998). "Jamming is not just cool any more." Nature, 396, 21-22.

Liu, C. (1994). "Spatial patterns of sound propagation in sand." Phys. Rev. B, 50.

Liu, C. and Nagel, S. R. (1992). "Sound in sand." Phys. Rev. Lett., 68(15), 2301-2304.

Liu, C. and Nagel, S. R. (1994). "Sound and vibration in granular materials." J. Phys.: Condens. Matter, 6, A433-A436.

Liu, C., Nagel, S. R., Schecter, D. A., Coppersmith, S. N., Majumdar, S., Narayan, O., and Witten, T. A. (1995). "Force fluctuations in bead packs." Science, 269, 513.

Liu, Y., Sun, W., Yuan, Z., and Fish, J. (2016). "A nonlocal multiscale discrete-continuum model for predicting mechanical behavior of granular materials." Int. J. Numer. Meth. Engng, 106, $129-160$.

Love, A. (1929). A Treatise of the Mathematical Theory of Elasticity. Cambridge University Pres.

M, Y., K, I., and W, V. (1998). "Effects of principal stress direction and intermediate principal stress on undrained shear behavior of san." Soils Found, 38, 179-188.

Magnanimo, V., La Ragione, L., Jenkins, J. T., Wang, P., and Makse, H. A. (2008). "Characterizing the shear and bulk moduli of an idealized granular material." Europ. Phys. Lett., 81, 34006.

Majmudar, T. and Behringer, R. (2005). "Contact force measurements and stress-induced anisotropy in granular materials." Nature, 435, 1079-1082.

Makse, H. A., Gland, N., Johnson, D., and Schwartz, L. (1999). "Why effective medium theory fails in granular materials." Phys. Rev. Lett., 83(24), 5070-5073.

Makse, H. A., Johnson, D. L., and Schwartz, L. M. (2000). "Packing of compressible granular materials." Phys. Rev. Lett., 84(18), 4160-4163.

Manzari, M. T. and Dafalias, Y. F. (1997). "A critical state two-surface plasticity model for sands." Géotechnique, 47, 255-272.

Masin, D. (2012). "Hypoplastic cam-clay model." Géotechnique, 62, 549-553.

Maugin, G. A. (1992). The Thermomechanics of Plasticity and Fracture. Cambridge University Press, Cambridge.

McNamara, S. and Young, W. R. (1992). "Inelastic collapse and clumping in a one-dimensional granular medium." Phys. Fluids A, 4(3), 496.

McNamara, S. and Young, W. R. (1994). "Inelastic collapse in two dimensions." Phys. Rev. E, 50(1), R28-R31. 
Mehrabadi, M. M. and Cowin, S. C. (1978). "Initial planar deformation of dilatant granular materials." J. Mech. Phys. Solids, 26, 269-284.

Metzger, P. T. (2004a). "Comment on "mechanical analog of temperature for the description of force distribution in static granular packings".." Phys Rev E Stat Nonlin Soft Matter Phys, 69(5 Pt 1), 053301; discussion 053302.

Metzger, P. T. (2004b). "Granular contact force density of states and entropy in a modified edwards ensemble.." Phys Rev E Stat Nonlin Soft Matter Phys, 70(5 Pt 1), 051303.

Miller, B., O'Hern, C., and Behringer, R. P. (1996). "Stress fluctuations for continously sheared granular materials." Phys. Rev. Lett., 77, 3110-3113.

Miller, T., Rognon, P., Metzger, B., and Einav, I. (2013). "Eddy viscosity in dense granular flows." Phys. Rev. Lett., 111, 058002.

Mitchell, J. K. and Soga, K. (2005). Fundamentals of Soil Behavior, third edition. Wiley.

Moreau, J. J. (1993). "New computation methods in granular dynamics." Powders \& Grains 93, Rotterdam, A. A. Balkema, 227.

Mroz, Z., Norris, V. A., and Zienkiewicz, O. C. (1978). "An anisotropic hardening model for soils and its application to cyclic loading." Int. J. Numer. Anal. Methods Geomech., 2, 203-221.

Mueth, D. M., Jaeger, H. M., and Nagel, S. R. (1998). "Force distribution in a granular medium." Phys. Rev. E, 57, 3164.

Mulhaus, H. B. and Vardoulakis, I. (1987). "The thickness of shear bands in granular materials." Géotechnique, 37, 271-283.

Nemat-Nasser, S., Mehrabadi, M. M., and Iwakuma, T. (1981). Three Dimensional Constitutive Relations and Ductile Fractures. North Holland, Amsterdam, Chapter On certain microscopic and macroscopic aspect of plastic flow of ductile material, 157-172.

Newland, P. L. and Allely, B. H. (1957). "Volume changes in drained taixial tests on granular materials." Géotechnique, 7, 17-34.

Nezamabadi, S., Radjai, F., Averseng, J., and Delenne, J.-Y. (2015). "Implicit frictional contact model for soft particle systems." Journal of the Mechanics and Physics of Solids, 83, 72-87.

Nguyen, D.-H., Azéma, E., Radjai, F., and Sornay, P. (2014). "Effect of size polydispersity versus particle shape in dense granular media." Physical Review E, 90(1), 012202.

Nichol, K., Zanin, A., Bastien, R., Wandersman, E., and van Hecke, M. (2010). "Flow-induced 
agitations create a granular fluid." Phys. Rev. Lett., 104, 078302.

Nicot, F. and Darve, F. (2007). "A micro-mechanical investigation of bifurcation." International Journal of Solids and Structures, 44, 6630-6652.

Nicot, F., Darve, F., and Khoa, H. (2007). "Bifurcation, second order-work in granular materials." International Journal for Numerical and Analytical Methods in Geomechanics, 31, 1007-1032.

Nitka, M., Combe, G., Dascalu, C., and Desrues, J. (2011). "Two-scale modeling of granular materials: a dem-fem approach." Granular Matter, 13, 277-281.

Noll, W. (1955). "Die herleitung der grundgleichungen der thermomechanik der kontinua aus der statistischen mechanik." J. Natural Mech. and Anal., XXX(4), 627-646.

Noll, W. (1958). "A mathematical theory of the mechanical behavior of continuous media." Arch. Ration. Mech. Anal, 2, 197-226.

Nova, R. (1982). Soil Mech. - transient Cycl. Load. Wiley, Chichester, Chapter A constitutive model for soil under monotonic and cyclic loading, 343-373.

Nova, R. (1994). "Controllability of the incremental response of soil specimens subjected to arbitrary loading programmes." Journal of the Mechanical Behavior of Materials, 5, 193-201.

Oda, M. (1972). "Initial fabrics and their relations to mechanical properties of granular material." Soils and foundations, 12, 17-36.

Oda, M., Iwashita, K., and Kakiuchi, T. (1997). "Importance of particle rotation in the mechanics of granular materials." Powders $\&$ Grains 1997, R. P. Behringer and J. T. Jenkins, eds., A. A. Balkema, Rotterdam, Netherlands, 207-210.

Oda, M., Konishi, J., and Nemat-Nasser, S. (1982). "Experimental micromechanical evaluation of strength of granular materials: effects of particle rolling." Mechanics of Materials, 1, 269-283.

Oda, M., Koshini, J., and Nemat-Nasser, S. (1980). "Some experimentally based fundamental results on the mechanical behavior of granular materials." Geotechnique, 30, 479-495.

O’Hern, C., Langer, S., Liu, A., and Nagel, S. (2001). "Force distributions near jamming and glass transitions.." Phys. Rev. Lett., 86(1), 111-114.

O’Hern, C., Silbert, L. E., Liu, A. J., and Nagel, S. R. (2003). "Jamming at zero temperature and zero applied stress: The epitome of disorder." Physical Review E, 68(1), 011306.

Oquendo, W. F., Munoz, J. D., and Radjai, F. (2016). "An equation of state for granular media at the limit state of isotropic compression." EPL, 114, 14004. 
Ouadfel, H. and Rothenburg, L. (2001). "Stress-force-fabric relationship for assemblies of ellipsoids." Mechanics of Materials, 33(4), 201-221.

Pavlovitch, A., Jullien, R., and Meakin, P. (1991). "Geometrical properties of a random packing of hard spheres." Physica A, 176, 206.

Peters, J. and Walizer, L. (2013). "Patterned non-affine motion in granular media." Journal of Engineering Mechanics, 139, 1479-1490.

Peyneau, P.-E. and Roux, J.-N. (2008a). "Frictionless bead packs have macroscopic friction, but no dilatancy." Physical Review E, 78.

Peyneau, P.-E. and Roux, J.-N. (2008b). "Solidlike behavior and anisotropy in rigid frictionless bead assemblies." Physical Review E, 78.

Pouliquen, O. (1999). "Scaling laws in granular flows down rough inclined planes." Phys. Fluids, $11(3), 542-548$.

Pouya, M. and Wan, R. (2016). "Strain in granular media: probabilistic approach to dirichlet tesselation." Journal of Engineering Mechanics.

Radjai, F. (2009). "Force and fabric states in granular media." Powders and Grains 2009, N. Masami and S. Luding, eds., AIP, New York, 35-42.

Radjai, F. (2015). "Modeling force transmission in granular materials." Comptes Rendus Physique, $16,3-9$.

Radjai, F., Delenne, J.-Y., Azema, É., and Roux, S. (2012). "Fabric evolution and accessible geometrical states in granular materials." Granular Matter, 14(2), 259-264.

Radjai, F., Jean, M., Moreau, J.-J., and Roux, S. (1996). "Force distributions in dense twodimensional granular systems." Phys. Rev. Lett., 77(2), 274-.

Radjai, F. and Richefeu, V. (2009). "Bond anisotropy and cohesion of wet granular materials." Phil. Trans. R. Soc. A, 367, 5123-5138.

Radjai, F. and Roux, S. (2002). "Turbulentlike fluctuations in quasistatic flow of granular media.." Phys Rev Lett, 89(6), 064302.

Radjai, F. and Roux, S. (2004). "Contact dynamics study of 2d granular media : Critical states and relevant internal variables." The Physics of Granular Media, H. Hinrichsen and D. E. Wolf, eds., Weinheim, Wiley-VCH, 165-186.

Radjai, F., Roux, S., and Moreau, J. J. (1999). "Contact forces in a granular packing.." Chaos, 
9(3), 544-550.

Radjai, F., Troadec, H., and Roux, S. (2004). "Key features of granular plasticity." Granular Materials: Fundamentals and Applications, S. Antony, W. Hoyle, and Y. Ding, eds., Cambridge, RS.C, 157-184.

Radjai, F. and Wolf, D. E. (1998). "The origin of static pressure in dense granular media." Granular Matter, 1, 3-8.

Radjai, F., Wolf, D. E., Jean, M., and Moreau, J. (1998). "Bimodal character of stress transmission in granular packings." Phys. Rev. Letter, 80, 61-64.

Reis, P. M., Jaeger, H. M., and van Hecke, M. (2015). "Designer matter: A perspective." Extreme Mechanics Letters, 5, 25-29.

Reynolds, O. (1885). "On the dilatancy of media composed of rigid particles in contact." Philos. Mag. Ser. 5, 50-20, 469.

Roscoe, K. H. (1970). "Tenth rankine lecture: The influence of strains in soil mechanics." Géotechnique, 20, 129-170.

Roscoe, K. H. and Schofield, A. N. (1963). "Mechanical behaviour of an idealised wet clay." 2nd Eur. Conf. Soil Mech. Found. Eng. Wiesbaden, 47-54.

Roscoe, K. H., Schofield, A. N., and Wroth, C. P. (1958). "On the yielding of soils." Géotechnique, $8,22-53$.

Roth, L. K. and Jaeger, H. M. (2016). "Optimizing packing fraction in granular media composed of overlapping spheres." Soft Matter, 12, 1107-1115.

Rothenburg, L. and Bathurst, R. J. (1989). "Analytical study of induced anisotropy in idealized granular materials." Geotechnique, 39, 601-614.

Roux, J.-N. (2000). "Geometric origin of mechanical properties of granular materials." Phys. Rev. E., 61, 6802-6836.

Roux, J.-N. and Combe, G. (2002). "Quasistatic rheology and the origins of strain." C. R. Physique, $3,131-140$.

Roux, S. and Radjai, F. (2001). "Statistical approach to the mechanical behavior of granular media." Mechanics for a New Millennium, H. Aref and J. Philips, eds., Netherlands, Kluwer Acad. Pub., $181-196$.

Rowe, P. W. (1962). "The stress-dilatancy relation for static equilibrium of an assembly of particles 
i contact." Proc. R. Soc. A Math. Phys. Eng. Sci. The Royal Societ, 500-527.

Russell, A. R., Wood, D. M., and Kikumoto, M. (2009). "Crushing of particles in idealized granular assemblies." J. Mech. Phys. Solids, 57, 1293-1313.

Saint-Cyr, B., Delenne, J.-Y., Voivret, C., Radjai, F., and Sornay, P. (2011). "Rheology of granular materials composed of nonconvex particles." Phys. Rev. E, 84(4), 041302-.

Satake, M. (1982). "Fabric tensor in granular materials." Proceedings of the IUTAM symposium on deformation and failure of granular materials, Delft, P. A. Vermeer and H. J. Luger, eds., Amsterdam, A. A. Balkema, 63-68.

Schofield, A. N. and Wroth, P. (1968). Critical State Soil Mechanics. McGraw-Hill, London.

Shibuya, S., Tatsuoka, F., Teachavorasinskun, S., Kong, X.-J., Abe, F., Kim, Y.-S., and Park, C.-S. (1992). "Elastic deformation properties of geomaterials." Soils and Foundations, 32, 26-46.

Silbert, L. E., Ertaş, D., Grest, G. S., Halsey, T. C., and Levine, D. (2002). "Geometry of frictionless and frictional sphere packings." Phys; Rev. E, 65(3), 031304.

Snoeijer, J. H., Vlugt, T. J. H., van Hecke, M., and van Saarloos, W. (2004). "Force network ensemble: a new approach to static granular matter.." Phys. Rev. Lett., 92(5), 054302.

Somfai, E., Roux, J.-N., Snoeijer, J. H., van Hecke, M., and van Saarloos, W. (2005). "Elastic wave propagation in confined granular systems." Phys. Rev. E, 72, 021301.

Somfai, E., van Hecke, M., Ellenbroek, W. G., Shundyak, K., and van Saarloos, W. (2007). "Critical and noncritical jamming of frictional grains." Phys. Rev. E, 75(2), 020301.

Staron, L. and Radjai, F. (2005). "Friction versus texture at the approach of a granular avalanche.." Phys Rev E, 72(4 Pt 1), 041308.

Staron, L., Radjai, F., and Vilotte, J.-P. (2005). "Multi-scale analysis of the stress state in a granular slope in transition to failure.." Eur Phys J E Soft Matter, 18(3), 311-320.

Staron, L., Vilotte, J.-P., and Radjai, F. (2002). "Preavalanche instabilities in a granular pile." Phys. Rev. Lett., 89, 204302.

Sun, J. and Sundaresan, S. (2011). "A constitutive model with microstructure evolution for flow of rate-independent granular materials." J. Fluid Mech., 682, 590-616.

Taboada, A., Estrada, N., and Radjaï, F. (2006). "Additive decomposition of shear strength in cohesive granular media from grain-scale interactions.." Phys. Rev. Lett., 97(9), 098302.

Taiebat, M. and Dafalias, Y. F. (2008). "Sanisand: Simple anisotropic sand plasticity model." Int. 
J. Numer. Anal. Meth. Geomech., 32, 915-948.

Taylor, D. W. (1948). Fundamentals of soil mechanics. Wiley, New York.

Tejchman, J. and Wu, W. (1993). "Numerical study on patterning of shear bands in a cosserat continuum." Acta Mechanica, 99, 61?74.

Terzaghi, K. (1943). Theoretical soil mechanics. J. Wiley, New York.

Thomann, T. G. and Hryciw, R. D. (1990). "Laboratory measurement of small strain shear modulus under $k_{0}$ conditions." ASTM Geotechnical Testing Journal, 13(2), 97-105.

Thornton, C. (1997). "Force transmission in granular media." KONA Powder and Particle, 15, $81-90$.

Thornton, C. and Randall, C. W. (1988). "Applications of theoretical contact mechanics to solid particle system simulation." Micromechanics of granular media, Amsterdam, Elsevier.

Tordesillas, A. (2007). "Force chain buckling, unjamming transitions and shear banding in dense granular assemblies." Philosophical Magazine, 87(32), 4987-5016.

Torquato, S. (2010). "Jammed hard-particle packings: From kepler to bernal and beyond." Reviews of Modern Physics, 82, 2633-2672.

Troadec, H., Radjai, F., Roux, S., and Charmet, J. (2002). "Model for granular texture with steric exclusion." Physical Review E, 66(4 1), 041305-1.

Vardoulakis, I. (1979). "Bifurcation analysis of the triaxial test on sand samples." Aeta Meehaniea, $32,35-54$

Vardoulakis, I. and Aifantis, E. C. (1991). "A gradient flow thoeory of plasticity for granular materials." Acta. Mech., 87, 197-217.

Vardoulakis, I. and Sulem, J. (1995). Bifurcation analysis in geomechanics. hapman \& Hall, London.

Verdugo, R. and Ishihara, K. (1996). "The steady state of sandy soils." Soils Found., 36, 81-91.

Vermeer, P. A. (1998). "Non-associated plasticity for soils, concrete and rock." Physics of Dry Granular Media, H. J. Herrmann, J.-P. Hovi, and S. Luding, eds., Dordrecht, Balkema, 163-196.

Voivret, C., Radjai, F., Delenne, J.-Y., and Youssoufi, M. E. (2009). "Force transmission in polydisperse granular media." Phys. Rev. Lett., 102, 178001.

Walton, K. (1987). "The effective elastic moduli of a random packing of spheres." Journal of Mechanics and Physics of Solids, 35, 213-226.

Walton, O. R. and Braun, R. L. (1986). "Viscosity, granular temperature, and stress calculations 
for shearing assemblies of inelastic, frictional disks." J. Rheol., 30, 949.

Wan, R. and Guo, P. (2004). "Stress dilatancy and fabric dependencies on sand behavior." Journal of Engineering Mechanics, 130, 635-645.

Wood, D. (1990). Soil behaviour and critical state soil mechanics. Cambridge University Press, Cambridge, England.

Wu, W. (1998). "Rational approach to anisotropy of sand." Int. J. Numer. Anal. Meth. Geomech., $22,921-940$.

Wu, W. and Niemunis, A. (1996). "Failure criterion, flow rule and dissipation function derived from hypoplasticit." Mech. Cohesive-Frict. Mater., 1, 145-163.

Wyart, M. (2006). "On the rigidity of amorphous solids." Annales de Physique Fr., 30, 1-96.

Wyart, M., Nagel, S. R., and Witten, T. A. (2005). "Geometric origin of excess low-frequency vibrational modes in weakly connected amorphous solids." Europhysics Letters, 72, 486-492.

Zhao, J. and Guo, N. (2013). "Unique critical state characteristics in granular media considering fabric anisotropy." Géotechnique, 63, 695-704.

Zhu, H., Mehrabadi, M. M., and Massoudi, M. (2006). "Incorporating the effects of fabric in the dilatant double shearing model for planar deformation of granular materials." International Journal of Plasticity, 22, 628-653.

Ziegler, H. and Wehrli, C. (1987). "The derivation of constitutive relations from the free energy and the dissipation function." Advances in Applied Mechanics, 25, 183-238. 


\section{List of Figures}

1 Evolution of stress ratio (a) and packing fraction (b) as a function of cumulative plastic shear strain $\varepsilon$ in DEM simulation of a packing of disks at constant confining pressure. Here, $q_{n}=0.5\left(\sigma_{1}-\sigma_{2}\right) \cos 2\left(\theta_{\sigma}-\theta_{0}\right)$ and $p=0.5\left(\sigma_{1}+\sigma_{2}\right)$, where $\sigma_{1}$ and $\sigma_{2}$ are the principal stresses and $\theta_{\sigma}$ and $\theta_{0}$ are the principal stress direction and flow direction, respectively. The initial state is prepared by isotropic compaction. Note the long transient and unmonotonic evolution of packing fraction upon shear reversal. 59

2 (a) The contact geometry; (b) First-shell particle environment with angular exclusions; (c) A loop of touching particles. . . . . . . . . . . . . . . . 60

3 (a) A map of normal forces in a polydisperse packing of disks. Line thickness is proportional to normal force; (b) A map of nonaffine particle displacements in a sheared packing of disks. . . . . . . . . . . . . . . . . . 61

4 Relevant scales in granular materials: contact, particle, assembly (microstructure), representative volume element (RVE) and macrostructure . . . . . . . . . . . . . 62

5 Shear modulus $G$ versus confining pressure $P$ in different types of numerically simulated, isotropically compressed glass bead assemblies, denoted as A (crosses, continuous line), B (asterisks, dotted line), C (square dots, continuous line) and D (open squares, dotted line). Corresponding solid fractions $\Phi$ and coordination numbers $z$ satisfy $\Phi_{A} \simeq \Phi_{C}>\Phi_{B}>\Phi_{D}$, and $z_{A}>z_{B}>z_{C} \simeq z_{D}$ The dashed line marked "KJ" corresponds to experimental data (Kuwano and Jardine 2002) on loose bead packs between 50 and $400 \mathrm{kPa} \ldots \ldots \ldots \ldots \ldots \ldots$

6 The packing fraction of an assembly of nonconvex agregates each composed of three overlapping disks as a function of the degree of nonconvexity $\eta \ldots \ldots$. . . . . . 64 

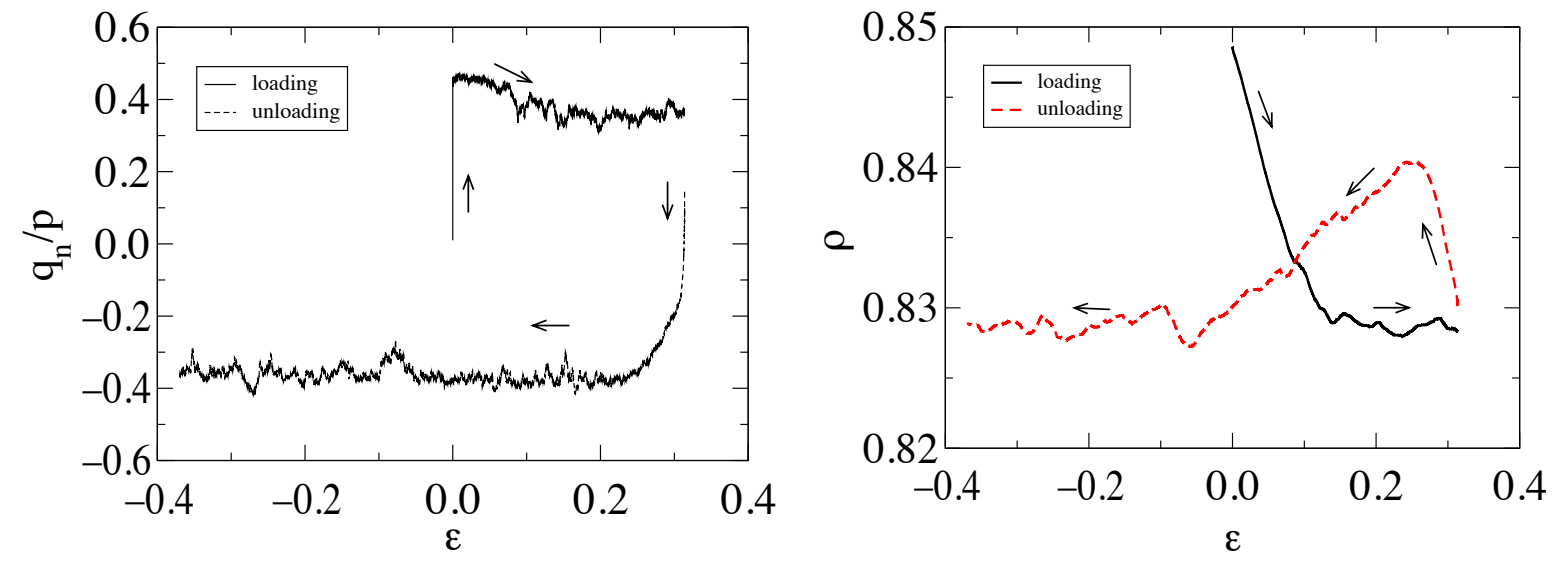

FIG. 1. Evolution of stress ratio (a) and packing fraction (b) as a function of cumulative plastic shear strain $\varepsilon$ in DEM simulation of a packing of disks at constant confining pressure. Here, $q_{n}=0.5\left(\sigma_{1}-\sigma_{2}\right) \cos 2\left(\theta_{\sigma}-\theta_{0}\right)$ and $p=0.5\left(\sigma_{1}+\sigma_{2}\right)$, where $\sigma_{1}$ and $\sigma_{2}$ are the principal stresses and $\theta_{\sigma}$ and $\theta_{0}$ are the principal stress direction and flow direction, respectively. The initial state is prepared by isotropic compaction. Note the long transient and unmonotonic evolution of packing fraction upon shear reversal. 


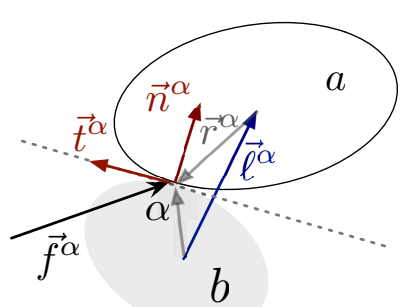

(a)

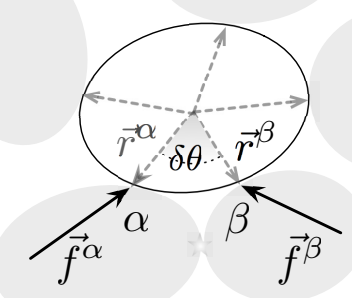

(b)

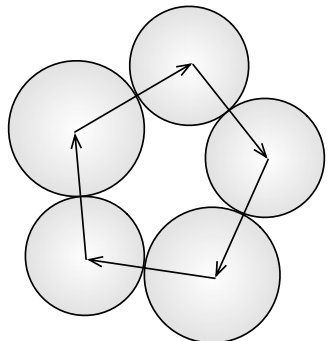

(c)

FIG. 2. (a) The contact geometry; (b) First-shell particle environment with angular exclusions; (c) A loop of touching particles. 

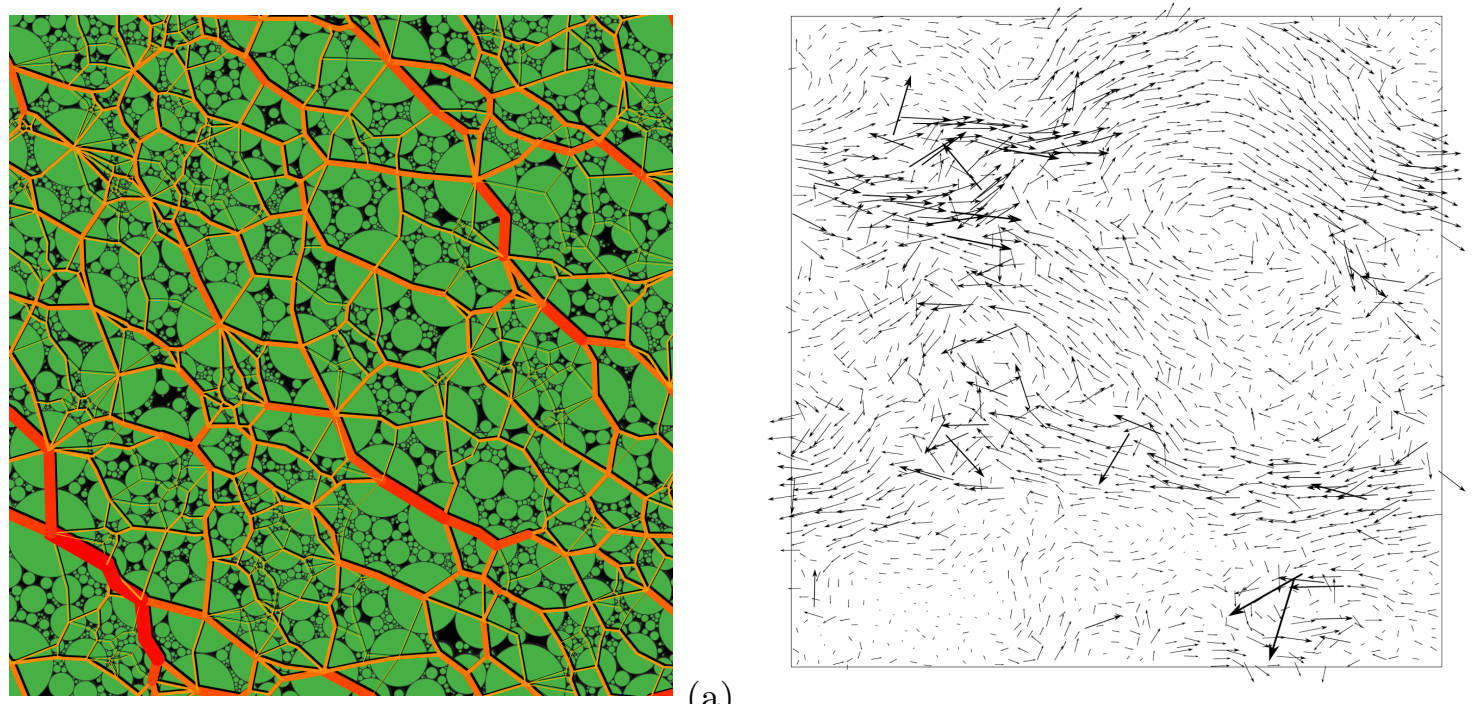

(a)

(b)

FIG. 3. (a) A map of normal forces in a polydisperse packing of disks. Line thickness is proportional to normal force; (b) A map of nonaffine particle displacements in a sheared packing of disks. 


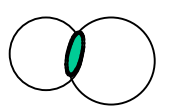

contact

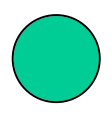

particle

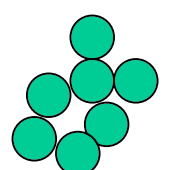

assembly

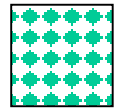

RVE

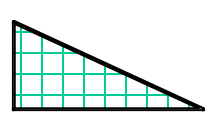

structure

FIG. 4. Relevant scales in granular materials: contact, particle, assembly (microstructure), representative volume element (RVE) and macrostructure. 


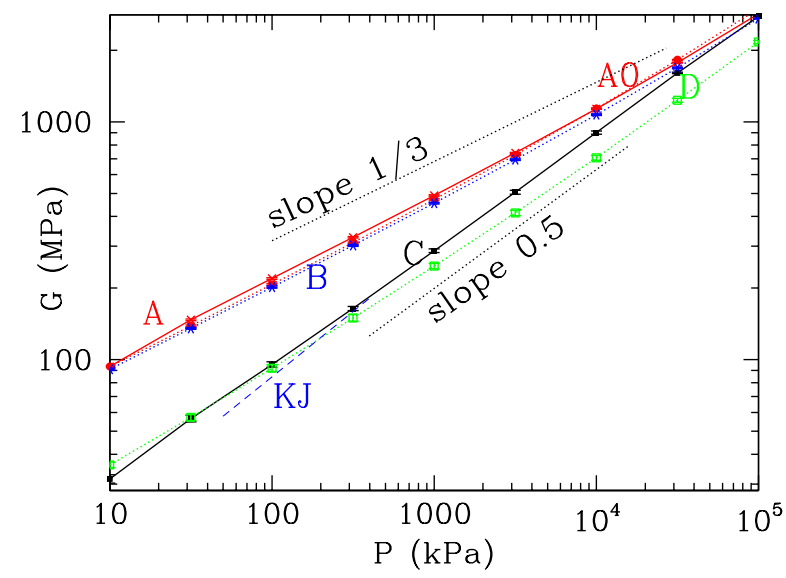

FIG. 5. Shear modulus $G$ versus confining pressure $P$ in different types of numerically simulated, isotropically compressed glass bead assemblies, denoted as A (crosses, continuous line), B (asterisks, dotted line), C (square dots, continuous line) and D (open squares, dotted line). Corresponding solid fractions $\Phi$ and coordination numbers $z$ satisfy $\Phi_{A} \simeq \Phi_{C}>\Phi_{B}>\Phi_{D}$, and $z_{A}>z_{B}>z_{C} \simeq z_{D}$ The dashed line marked " $\mathrm{KJ}$ " corresponds to experimental data (Kuwano and Jardine 2002) on loose bead packs between 50 and $400 \mathrm{kPa}$. 


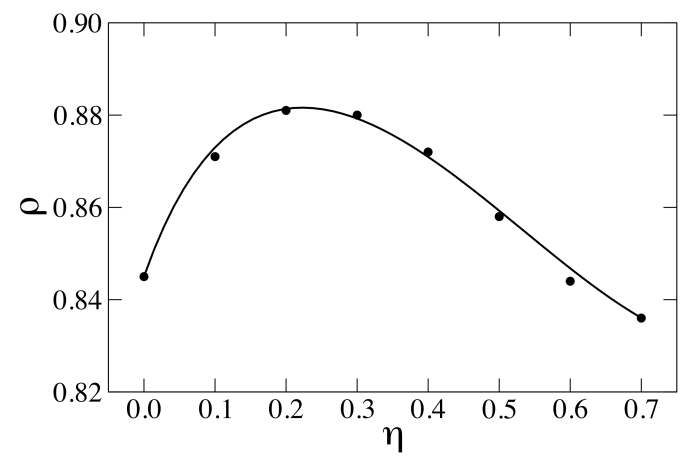

FIG. 6. The packing fraction of an assembly of nonconvex agregates each composed of three overlapping disks as a function of the degree of nonconvexity $\eta$. 